\title{
FRACTIONAL POWERS OF GENERATORS OF EQUICONTINUOUS SEMIGROUPS AND FRACTIONAL DERIVATIVES
}

\author{
OSCAR E. LANFORD III and DEREK W. ROBINSON
}

(Received 21 September 1987)

Communicated by I. Raeburn

\begin{abstract}
We analyze fractional powers $H^{\alpha}, \alpha>0$, of the generators $H$ of uniformly bounded locally equicontinuous semigroups $S$. The $H^{\alpha}$ are defined as the $\alpha$ th derivative $\delta^{\alpha}$ of the Dirac measure $\delta$ evaluated on $S$. We demonstrate that the $H^{\alpha}$ are closed operators with the natural properties of fractional powers, for example, $H^{\alpha} H^{\beta}=H^{\alpha+\beta}$ for $\alpha, \beta>0$, and $\left(H^{\alpha}\right)^{\beta}=H^{\alpha \beta}$ for $1>\alpha>0$ and $\beta>0$. We establish that $H \alpha$ can be evaluated by the Balakrishnan-Lions-Peetre algorithm

$$
H^{\alpha} x=\lim _{\varepsilon \rightarrow 0} c_{\alpha, m}^{-1} \int_{\varepsilon}^{\infty} \frac{d t}{t} \frac{\left(I-S_{\ell}\right)^{m}}{t^{\alpha}} x
$$

where $m$ is an integer larger than $\alpha, c_{\alpha, m}$ is a suitable constant, and the limit exists in the appropriate topology if, and only if, $x \in D\left(H^{\alpha}\right)$. Finally we prove that $H^{\alpha}$ is the fractional derivative of $S$ in the sense

$$
H^{\alpha} x=\lim _{t \rightarrow 0+}\left(\left(I-S_{t}\right) / t\right)^{\alpha} x
$$

where the limit again exists if, and only if, $x \in D\left(H^{\alpha}\right)$.

1980 Mathematics subject classification (Amer. Math. Soc.) (1985 Revision): 47 A 99.
\end{abstract}

\section{Introduction}

The operator $D$ of differentiation acts through multiplication by $-i \lambda$ in Fourier space, where $\lambda$ is the Fourier variable. Hence, for $\alpha>0$, one can define the fractional power $D^{\alpha}$ of differentiation as multiplication by $(-i \lambda)^{\alpha}$. But multiplication by $(-i \lambda)^{\alpha}$ corresponds to convolution of the inverse Fourier transform with a distribution $\delta^{\alpha}$, the fractional derivative of

(C) 1989 Australian Mathematical Society 0263-6115/89 $\$ A 2.00+0.00$ 
the Dirac measure $\delta$. Hence $D^{\alpha}$ can be interpreted as the distribution $\delta^{\alpha}$ evaluated on the group $S_{t}=\exp \{-t D\}$ of translations, that is, as the operator $S_{\delta^{\prime \prime}}$ with action

$$
\begin{aligned}
\left(S_{\delta^{n}} f\right)(x) & =\int d y \delta^{\alpha}(y)\left(S_{y} f\right)(x) \\
& =\int d y \delta^{\alpha}(y) f(x-y) \\
& =\left(\delta^{\alpha} * f\right)(x)=\left(D^{\alpha} f\right)(x) .
\end{aligned}
$$

The aim of this paper is to analyze fractional powers of generators of uniformly bounded equicontinuous semigroups by an analogous method. Specifically if $S_{t}=\exp \{-t H\}$ is a uniformly bounded strongly continuous semigroup acting on the Banach spaces $\mathscr{X}$ then we define $H^{\alpha}$ by

$$
H^{\alpha} x=\lim _{\eta} S_{\delta^{*} * \eta} x
$$

where $D\left(H^{\alpha}\right)$ consists of those $x \in \mathscr{Z}$ such that the strong limit, over an appropriate net of test functions $\eta$, exists. This method of defining $H^{\alpha}$ has several advantages. It is quite intuitive and agrees with the standard method of defining fractional derivatives. It readily extends to generators of a large class of equicontinuous semigroups which induces strongly continuous or weakly* continuous semigroups. It leads to easy proofs of basic properties such as multiplicativity, that is, $H^{\alpha} H^{\beta}=H^{\alpha+\beta}$. It differs, however, from the traditional methods of defining fractional powers by integral algorithms. Nevertheless one of our principal conclusions is that it gives the same results as the traditional theory.

The standard theory of fractional powers of generators originated with the early work of Bochner [5] and Phillips [17] on convolution semigroups but then developed along quite different lines than those sketched above. Balakrishran [2] defined the fractional powers $H^{\alpha}, 0<\alpha<1$, by the formula

$$
H^{\alpha} x=\frac{\alpha}{\Gamma(1-\alpha)} \int_{0}^{\infty} \frac{d t}{t} \frac{\left(I-S_{t}\right)}{t^{\alpha}} x
$$

with $D\left(H^{\alpha}\right)$ taken to be the set of $x \in \mathscr{X}$ for which the integral converges. Similar or equivalent definitions were independently proposed by Kato [10], Nelson [15], and Krasnoselskii and Sobolevskii [12]. Somewhat later Lions and Peetre [14] proved that if $\alpha$ is a positive integer then

$$
H^{\alpha} x=\frac{1}{c_{\alpha, m}} \int_{0}^{\infty} \frac{d t}{t} \frac{\left(I-S_{t}\right)^{m}}{t^{\alpha}} x
$$

where $m$ is an integer strictly larger than $\alpha$ and

$$
c_{\alpha, m}=\int_{0}^{\infty} \frac{d t}{t} \frac{\left(1-e^{-t}\right)^{m}}{t^{\alpha}} .
$$


Subsequently this algorithm has been used as the definition of $H^{\alpha}$ for all $\alpha>0$. Many other authors contributed to the study of fractional powers of generators through the above integral algorithms or alternative algorithms using powers of the resolvent of $H$, and summaries of the subject from various aspects, at various stages of its development, with extensive references and applications, can be found in the books of Yosida [22], Friedman [9], Krasnoselskii et al [13], Triebel [20], Tanabe [19], and Pazy [16]. A comprehensive description of the subject is also given in the series of papers by Komatsu [11]. Most of this work concerns generators of strongly continuous semigroups, or operators with strongly continuous resolvent families, but Berens, Butzer, Westphal [3] and Komatsu [11] have also derived results in the case of weak* continuity. Our methods have the advantage of unifying these two cases, and extending the results in several ways.

The starting point of our investigation is Schwartz' definition [18] of the operator $S_{\varphi}$ associated with a tempered distribution $\varphi$ evaluated on an equicontinuous semigroup $S$. There are various possible formulations of the semigroup theory but we have chosen to follow that of Arveson [1]. This theory is outlined in Section 2 where a number of preliminary equicontinuity properties are discussed. In Section 3, following Schwartz [18], we define $S_{\varphi}$ as a limit over a net of test functions $\eta$ of the bounded operators

$$
S_{\varphi * \eta}=\int_{0}^{\infty} d t(\varphi * \eta)(t) S_{t}
$$

A number of elementary properties of the $S_{\varphi}$, their products, and the map $\varphi \mapsto S_{\varphi}$ are also derived. Then in Section 4 we discuss the Fourier theory of various special measures and distributions, for example the distributions $\delta^{\alpha}$. After these preliminaries we begin the analysis of the fractional powers $H^{\alpha}$, $\alpha>0$, of the semigroup generator $H$, in Section 5 .

If $S_{t}=\exp \{-t H\}$ then $H^{\alpha}$ is defined as $S_{\delta^{\alpha}}$. Starting from this definition the integral algorithm $(*)$ corresponds to an approximation

$$
H^{\alpha} x=S_{\delta^{\alpha}} x=\lim _{\varepsilon \rightarrow 0} S_{\varphi_{\alpha, \varepsilon}} x
$$

where the $\varphi_{\alpha, \varepsilon}$ are bounded measures on $[0, \infty)$. Specifically $\varphi_{\alpha, \varepsilon}$ is the Fourier transform of the function

$$
\lambda \in \mathbf{R} \mapsto \frac{(-i)^{\alpha}}{c_{\alpha, m}} \int_{\varepsilon}^{\infty} \frac{d t}{t} \frac{\left(1-e^{i \lambda t}\right)^{m}}{t^{\alpha}}
$$

and (**) is a reflection of the fact that $\varphi_{\alpha, \varepsilon} * \eta \rightarrow \delta^{\alpha} * \eta$ in $L_{1}(0, \infty)$ for each test function $\eta$ with compact support. This is only one of many possible $L_{1}$ approximants to $\delta^{\alpha}$ which lead to a representation of $H^{\alpha}$ by regularization with $S$. 
Finally, in Section 6, we use this freedom of approximation to establish a different characterization of $H^{\alpha}$. We prove that the fractional power $H^{\alpha}$ can be identified as the fractional derivative of the semigroup $S$, that is

$$
H^{\alpha} x=\lim _{t \rightarrow 0}\left(\left(1-S_{t}\right) / t\right)^{\alpha} x
$$

with convergence if, and only if, $x \in D\left(H^{\alpha}\right)$.

\section{Continuous semigroups}

Let $\mathscr{X}$ denote a Banach space with norm $\|\cdot\|$ and $\mathscr{F}$ a norm-closed subspace of the dual $\mathscr{X}^{*}$ of $\mathscr{X}$. Further let $\sigma(\mathscr{Z}, \mathscr{F})$ be the locally convex topology on $\mathscr{Z}$ induced by the functionals in $\mathscr{F}$. We assume

1. $\|x\|=\sup \{|f(x)| ; f \in \mathscr{F},\|f\| \leq 1\}$,

2. the $\sigma(\mathscr{X}, \mathscr{F})$-closed convex hull of every $\sigma(\mathscr{X}, \mathscr{F})$-compact set in $\mathscr{X}$ is $\sigma(\mathscr{Z}, \mathscr{F})$-compact,

3. the $\sigma(\mathscr{F}, \mathscr{Q})$-closed convex hull of every $\sigma(\mathscr{F}, \mathscr{Z})$-compact set in $\mathscr{F}$ is $\sigma(\mathscr{F}, \mathscr{Z})$-compact.

These conditions are satisfied if $\mathscr{F}=\mathscr{X}^{*}$, or if $\mathscr{Z}$ has a predual $\mathscr{X}_{*}$ and $\mathscr{F}=\mathscr{X}_{*}$ (see [1] or Chapter 3 of [6]), and they appear to be the minimum requirements for the development of a satisfactory theory of integration for semigroups continuous with respect to the $\sigma(\mathscr{Z}, \mathscr{F})$-topology. We note that Arveson (private communication) has established that conditions 2 and 3 are not a consequence of condition 1 and the fact that $\mathscr{F}$ is norm-closed.

Next we define a $\sigma(\mathscr{Z}, \mathscr{F})$-continuous semigroup to be a semigroup $S=$ $\left\{S_{l}\right\}_{t \geq 0}$ of bounded linear operators on $\mathscr{Z}$ such that

1. $t \in \mathbf{R}_{+} \mapsto S_{t} x \in \mathscr{X}$ is $\sigma(\mathscr{X}, \mathscr{F})$-continuous for all $x \in \mathscr{X}$, that is, $t \mapsto f\left(S_{t} x\right)$ is continuous for all $x \in \mathscr{X}$ and $f \in \mathscr{F}$.

2. $x \in \mathscr{Z} \mapsto S_{t} x \in \mathscr{Z}$ is $\sigma(\mathscr{X}, \mathscr{F})-\sigma(\mathscr{X}, \mathscr{F})$-continuous for all $t \geq 0$, that is, $f \circ S_{t} \in \mathscr{F}$ for all $t \geq 0$ and $f \in \mathscr{F}$.

There are two basic properties of a $\sigma(\mathscr{Z}, \mathscr{F})$-continuous semigroup $S$. First there exist an $M \geq 1$ and $\omega \geq 0$ such that

$$
\left\|S_{t}\right\| \leq M e^{\omega t}
$$

for all $t \geq 0$. In particular $S$ is called a uniformly bounded semigroup if such bounds hold with $\omega=0$. Second if $\mu$ is a complex measure on $\mathbf{R}_{+}$satisfying $\int_{0}^{\infty} d|\mu|(t) e^{\omega t}<\infty$ then

$$
S_{\mu} x=\int_{0}^{\infty} d \mu(t) S_{t} x
$$

defines a bounded $\sigma(\mathscr{X}, \mathscr{F})-\sigma(\mathscr{X}, \mathscr{F})$-continuous operator $S_{\mu}$ on $\mathscr{X}$. 
Now that if $\eta \in C_{00}^{\infty}\left(\boldsymbol{R}_{+}\right)$, the infinitely-often differentiable functions with compact support in $(0, \infty)$, we also write

$$
S_{\eta}=\int d t \eta(t) S_{t}
$$

for the associated bounded operator. Then the set $\mathscr{X}_{\text {reg }}(S)$ of regularized elements is defined by

$$
\mathscr{X}_{\text {reg }}(S)=\left\{S_{\eta} x ; x \in \mathscr{X}, \eta \in C_{00}^{\infty}\left(\mathbf{R}_{+}\right)\right\} .
$$

Next we introduce the $(\sigma(\mathscr{X}, \mathscr{F})$-) $)$ generator of $S$ as the $\sigma(\mathscr{X}, \mathscr{F})$-right derivative of $S$ at the origin. Explicitly $D(H)$ consists of those $x \in \mathscr{Z}$ for which there exists a $y \in \mathscr{Z}$ with the property $f(y)=\lim _{t \rightarrow 0+} f\left(\left(I-S_{t}\right) x\right) / t$ for all $f \in \mathscr{F}$. Then the action of $H$ is given by $H x=y$.

Note that the semigroup property of $S$ automatically implies $S_{t} D(H) \subseteq$ $D(H)$ and $H S_{t} x=S_{t} H x$ for all $x \in D(H)$. Moreover one has the identity

$$
\left(I-S_{t}\right) x=\int_{0}^{t} d s S_{s} H x
$$

for all $x \in D(H)$ and $t \geq 0$. The basic properties of the generator $H$ and its powers $H^{n}$ are summarized in the next two propositions.

Proposition 2.1. Let $S$ be a $\sigma(\mathscr{X}, \mathscr{F})$-continuous semigroup with generator $H$. Then

1. the operators $H^{n}, n=1,2, \ldots$, are $\sigma(\mathscr{X}, \mathscr{F})-\sigma(\mathscr{X}, \mathscr{F})$-closed,

2. the regularized elements $\mathscr{X}_{\text {reg }}(S)$ form a $\sigma(\mathscr{X}, \mathscr{F})$-dense common core of the $H^{n}$.

Proof. 1. Assume $x_{j} \in D(H)$ and $x_{j} \rightarrow x, H x_{j} \rightarrow y$, in the $\sigma(\mathscr{X}, \mathscr{F})-$ topology. Then it follows from the $\sigma(\mathscr{X}, \mathscr{F})$-continuity of $S$ and the identity (*) that

$$
\left(I-S_{t}\right) x=\int_{0}^{t} d s S_{s} y
$$

But this immediately implies that $x \in D(H)$ and $H x=y$, that is, $H$ is $\sigma(\mathscr{X}, \mathscr{F})-\sigma(\mathscr{X}, \mathscr{F})$-closed. Now we proceed by induction. Suppose $H^{m}$, $m=1,2, \ldots, n-1$, are $\sigma(\mathscr{X}, \mathscr{F})-\sigma(\mathscr{X}, \mathscr{F})$-closed operators. Then if $x \in$ $D\left(H^{n}\right)$ it follows that $S_{s} H^{n-1} x \in D(H)$ and since $H$ is closed

$$
\int_{0}^{t} d s(t-s)^{n-1} S_{s} H^{n-1} x \in D(H) .
$$

Moreover

$$
H \int_{0}^{t} d s \frac{(t-s)}{(n-1) !}^{n-1} S_{s} H^{n-1} x=(-1)^{n}\left(I-S_{t}\right) x+\sum_{m=1}^{n-1}(-1)^{m} \frac{t^{n-m}}{(n-m) !} H^{n-m} x
$$


by a straightforward calculation. Considering this identity for $n-1$ distinct values $t_{1}, t_{2}, \ldots, t_{n-1}$, one concludes by linear algebra that each $H^{m} x, m=$ $1,2, \ldots, n-1$, can be expressed as a linear combination of $x$ and $H^{n} x$. Thus if $x_{j} \in D\left(H^{n}\right)$ and $x_{j} \rightarrow x, H^{n} x_{j} \rightarrow y$, in the $\sigma(\mathscr{X}, \mathscr{F})$-topology then each $H^{m} x_{j}$ is $\sigma(\mathscr{X}, \mathscr{F})$-convergent. But the operators $H^{m}, m=1,2, \ldots, n-1$, are $\sigma(\mathscr{X}, \mathscr{F})-\sigma(\mathscr{Z}, \mathscr{F})$-closed by assumption and hence $H^{m} x_{j} \rightarrow H^{m} x$. Finally one has the identities

$$
\left(I-S_{t}\right) H^{n-1} x_{j}=\int_{0}^{t} d s S_{s} H^{n} x_{j}
$$

and in the $\sigma(\mathscr{X}, \mathscr{F})$-limit one obtains

$$
\left(I-S_{t}\right) H^{n-1} x=\int_{0}^{t} d s S_{s} y
$$

because of the foregoing argument. It follows immediately that $x \in D\left(H^{n}\right)$ and $H^{n} x=y$. Thus $H^{n}$ is $\sigma(\mathscr{X}, \mathscr{F})-\sigma(\mathscr{X}, \mathscr{F})$-closed and the proof is complete by induction

2. If $y=S_{\eta} x \in \mathscr{L}_{\text {reg }}(S)$ then $y \in D(H)$ and $H y=S_{\eta^{\prime}} x$. Then by iteration $y \in D\left(H^{n}\right)$ and $H^{n} y=S_{\eta^{(n)}} x$. Now replacing $\eta$ by $\eta_{m}$ where $\eta_{m}(t)=m \eta(m t)$ one has $S_{\eta_{m}} x=\int d t \eta(t) S_{t / m} x$. Therefore $S_{\eta_{m}} x \rightarrow \int d t \eta(t) x$ in the $\sigma(\mathscr{Z}, \mathscr{F})$ toplogy as $m \rightarrow \infty$. Moreover if $x \in D\left(H^{n}\right)$ then

$$
H^{n} S_{\eta_{m}} x=S_{\eta_{m}} H^{n} x \rightarrow \int d t \eta(t) x .
$$

This establishes that $\mathscr{X}_{\text {reg }}(S)$ is a $\sigma(\mathscr{X}, \mathscr{F})$-dense subspace of $X$ and that it is a core for each $H^{n}$.

The next proposition gives the basic properties of the resolvent of the generator.

Proposition 2.2. Let $S$ be a $\sigma(\mathscr{X}, \mathscr{F})$-continuous semigroup with generator $H$ satisfying $\left\|S_{t}\right\| \leq M e^{\omega t}, t \geq 0$. It follows that if $\operatorname{Re} \mu>\omega$ then $\mu \in r(-H)$ the resolvent set of $-H$, that is, the inverse operator $(\mu I+H)^{-1}$ exists as a bounded $\sigma(\mathscr{Z}, \mathscr{F})-\sigma(\mathscr{X}, \mathscr{F})$-continuous operator. Moreover

$$
(\mu I+H)^{-n} x=\int_{0}^{\infty} d s \frac{s^{n-1}}{(n-1) !} e^{-\mu s} S_{s} x
$$

Proof. First define the bounded $\sigma(\mathscr{X}, \mathscr{F})-\sigma(\mathscr{X}, \mathscr{F})$-continuous operators $R_{n}(\mu)$ on $\mathscr{Z}$ by

$$
R_{n}(\mu) x=\int_{0}^{\infty} d s \frac{s^{n-1}}{(n-1) !} e^{-\mu s} S_{s} x
$$

Then an elementary calculation establishes that $R_{n}(\mu) x \in D(H)$ for any $x \in \mathscr{Z}$ and $(\mu I+H) R_{n}(\mu) x=R_{n-1}(\mu) x$. Similarly if $x \in D(H)$ then 
one calculates that $R_{n}(\mu)(\mu I+H) x=R_{n-1}(\mu) x$. It follows immediately that $R_{1}(\mu)=(\mu I+H)^{-1}$ and $R_{n}(\mu)=(\mu I+H)^{-1} R_{n-1}(\mu)=(\mu I+H)^{-n}$.

The Mackey topology $\tau(\mathscr{Z}, \mathscr{F})$ is also essential in the subsequent discussion. This topology is defined by the family of seminorms

$$
x \in \mathscr{Z} \mapsto p_{K}(x)=\sup _{f \in K}|f(x)|
$$

where $K$ ranges over the $\sigma(\mathscr{X}, \mathscr{F})$-compact subsets of $\mathscr{F}$. Usually the Mackey topology is defined in terms of the convex, compact, circled, subset of $\mathscr{F}$ but this is equivalent to the foregoing definition because we are assuming that the $\sigma(\mathscr{X}, \mathscr{F})$-closed convex hull of a $\sigma(\mathscr{Z}, \mathscr{F})$-compact set is again $\sigma(\mathscr{Z}, \mathscr{F})$ compact. The $\tau(\mathscr{X}, \mathscr{F})$-topology on $\mathscr{Z}$ is in fact the finest locally convex topology such that all $\tau(\mathscr{X}, \mathscr{F})$-continuous functionals lie in $\mathscr{F}$. In particular any $\sigma(\mathscr{X}, \mathscr{F})$-dense convex subset is $\tau(\mathscr{X}, \mathscr{F})$-dense. The $\tau(\mathscr{X}, \mathscr{F})$-topology is particularly simple if $\mathscr{F}=\mathscr{X}^{*}$. The unit ball of $\mathscr{X}^{*}$ is $\sigma\left(\mathscr{X}^{*}, \mathscr{X}\right)$-compact, by the Alaoglu-Bourbaki theorem and hence the $\tau\left(\mathscr{X}, \mathscr{X}^{*}\right)$-topology is equal to the norm topology.

Next we define a family $\left\{T_{\alpha}\right\}$ of bounded operators on $\mathscr{X}$ to be $(\tau(\mathscr{X}, \mathscr{F})-)$ equicontinuous if for each compact subset $K \subseteq \mathscr{F}$ there exist a compact subset $K^{\prime}$ such that

$$
p_{K}\left(T_{\alpha} x\right) \leq p_{K}^{\prime}(x)
$$

for all $x \in \mathscr{Z}$ and for all $\alpha$. In particular the semigroup $S$ is equicontinuous if the family $\left\{S_{t}\right\}_{t \geq 0}$ satisfies this property. More generally $S$ is defined to be locally $\left(\tau(\mathscr{Z}, \mathscr{F})\right.$-)equicontinuous if the family $\left\{S_{t}\right\}_{t \in\left[0, t_{0}\right]}$ is equicontinuous for each $t_{0} \in[0, \infty)$, or, equivalently, for $t_{0}=1$.

Proposition 2.3. Let $S$ be a $\sigma(\mathscr{Z}, \mathscr{F})$-continuous semigroup with $\sigma(\mathscr{X}, \mathscr{F})$-generator $H$. If $S$ is locally $\tau(\mathscr{X}, \mathscr{F})$-equicontinuous then it is $\tau(\mathscr{Z}, \mathscr{F})$-continuous and the $\tau(\mathscr{Z}, \mathscr{F})$-generator of $S$ is equal to $H$, that is, for each $x \in D(H)$

$$
\lim _{t \rightarrow 0+}\left(I-S_{t}\right) x / t=H x
$$

where the limit is in the $\tau(\mathscr{X}, \mathscr{F})$-topology.

Proof. If $x \in D(H)$ then

$$
f\left(S_{s} x-S_{t} x\right)=\int_{s}^{t} d r f\left(S_{r} H x\right)
$$

and hence $p_{K}\left(S_{s} x-S_{t} x\right) \leq p_{K^{\prime}}(H x)|t-s|$ for $0 \leq t, s \leq 1$. Now for $x \in D(H)$ and $y \in \mathscr{Z}$ one has

$$
\begin{aligned}
p_{K}\left(S_{s} y-S_{t} y\right) & \left.\leq p_{K}\left(S_{s} x-S\right)_{t} x\right)+p_{K}\left(S_{s}(y-x)\right)+p_{K}\left(S_{t}(y-x)\right) \\
& \leq p_{K^{\prime}}(H x)|s-t|+2 p_{K^{\prime}}(y-x)
\end{aligned}
$$


for $0 \leq s, t \leq 1$. But $D(H)$ is a $\sigma(\mathscr{X}, \mathscr{F})$-dense subspace of $\mathscr{X}$ and hence it is $\tau(\mathscr{X}, \mathscr{F})$-dense. Consequently this estimate implies $\tau(\mathscr{Z}, \mathscr{F})$-continuity.

Finally if $x \in D(H)$ then

$$
\left(I-S_{t}\right) x / t=t^{-1} \int_{0}^{t} d s S_{s} H x .
$$

But the $\tau(\mathscr{X}, \mathscr{F})$-limit of the right hand side exists as $t \rightarrow 0+$ because of the $\tau(\mathscr{X}, \mathscr{F})$-continuity of $S$ and hence the $\tau(\mathscr{X}, \mathscr{F})$-limit of the left hand side also exists.

There are two important examples in which local equicontinuity of a $\sigma(\mathscr{X}, \mathscr{F})$-continuous semigroup $S$ is automatic. If $\mathscr{F}=\mathscr{X}^{*}$ or if $\mathscr{Z}$ has a predual $\mathscr{Z}_{*}$ and $\mathscr{F}=X_{*}$.

First, if $\mathscr{F}=\mathscr{Z}^{*}$ then the $\tau(\mathscr{Z}, \mathscr{F})$-topology and the norm topology are equal and local equicontinuity of the $\sigma\left(\mathscr{X}, \mathscr{X}^{*}\right)$-continuous semigroup $S$ follows from the bounds $\left\|S_{t}\right\| \leq M \exp \{\omega t\}$.

Second, if $\mathscr{F}=\mathscr{X}_{*}$ then the dual semigroup $S^{*}=\left\{S_{t}^{*}\right\}_{t \geq 0}$ formed by the duals (adjoints) $S_{t}^{*}$ on $\mathscr{X}_{*}$ of the operators $S_{t}$ on $\mathscr{X}$ form a $\sigma\left(\mathscr{X}_{*}, \mathscr{X}\right)$ continuous semigroup. But $\sigma\left(\mathscr{Z}_{*}, \mathscr{X}\right)$-continuity is equivalent to strong continuity and hence $(t, f) \in[0,1] \times \mathscr{X}_{*} \mapsto S_{t}^{*} f \in \mathscr{Z}_{*}$ is jointly continuous on bounded subsets of $\mathscr{X}_{*}$. Therefore $S$ is locally $\tau\left(\mathscr{X}, \mathscr{X}_{*}\right)$-equicontinuous by duality. A more general result of this type is given by the following proposition

Proposition 2.4. Let $S$ be a $\sigma(\mathscr{Z}, \mathscr{F})$-continuous semigroup. Then the following conditions are equivalent:

1. $S$ is locally $\tau(\mathscr{Z}, \mathscr{F})$-equicontinuous.

2. For each $\sigma(\mathscr{F}, \mathscr{Z})$-compact subset $K$ the set

$$
\bigcup_{0 \leq t \leq 1} S_{t}^{*} K
$$

is $\sigma(\mathscr{F}, \mathscr{X})$-precompact.

3. The map $(t, f) \in[0,1] \times \mathscr{F} \mapsto S_{t}^{*} f \in \mathscr{F}$ is jointly continuous on bounded subsets of $\mathscr{F}$.

Remark 2.5. The implications $1 \Leftrightarrow 2 \Leftarrow 3$ are valid for any family $S$ of bounded $\sigma(\mathscr{X}, \mathscr{F})$-continuous operators. The semigroup property is irrelevant. The equivalence $1 \Leftrightarrow 2$ follows by duality and the implication $3 \Rightarrow 2$ is straightforward.

Proof. In the light of the foregoing remark it suffices to prove $1 \Rightarrow 3$.

Assume $t_{n} \rightarrow t, 0 \leq t_{n} \leq 1, f_{n} \rightarrow 0$ in the $\sigma(\mathscr{F}, \mathscr{Z})$-topology, and $\left\|f_{n}\right\| \leq 1$. We must show that $S_{t_{n}}^{*} f_{n}$ is $\sigma(\mathscr{F}, \mathscr{Z})$-convergent to zero or, equivalently, 
$\left.\lim _{n \rightarrow \infty} f_{n}\left(\left(S_{t_{n}}-S_{t}\right) x\right)\right)=0$ for all $x \in \mathscr{Z}$. Now let $K$ denote the $\sigma(\mathscr{F}, \mathscr{Z})$ compact set $\left\{f_{n}\right\}_{n \geq 1} \cup\{0\}$ and choose $K^{\prime}$ such that $p_{K}\left(S_{s} x\right) \leq p_{K^{\prime}}(x)$ for all $0 \leq s \leq 1$ and all $x \in \mathscr{Z}$. Next remark that the domain $D(H)$ of the generator $H$ of $S$ is $\sigma(\mathscr{X}, \mathscr{F})$-dense and hence $\tau(\mathscr{Z}, \mathscr{F})$-dense. Thus given $x \in \mathscr{Z}$ and $\varepsilon>0$ one may choose $y \in D(H)$ such that $p_{K^{\prime}}(x-y)<\varepsilon / 2$. Therefore $\left|f_{n}\left(S_{s}(x-y)\right)\right|<\varepsilon / 2$ for all $0 \leq s \leq 1$ and all $n \geq 1$. Consequently

$$
\begin{aligned}
\left.\mid f_{n}\left(\left(S_{t_{n}}-S_{t}\right) x\right)\right) \mid & \left.\leq \mid f_{n}\left(\left(S_{t_{n}}-S_{t}\right) y\right)\right) \mid+\varepsilon \\
& \leq\left|\int_{t_{n}}^{t} d s f_{n}\left(S_{s} H y\right)\right|+\varepsilon \\
& \leq\left|t-t_{n}\right||H y|+\varepsilon .
\end{aligned}
$$

The desired conclusion follows immediately.

Next we use this characterization of local equicontinuity to examine equicontinuity of families $\left\{S_{\mu}\right\}$ where $\mu$ are measures on $\mathbf{R}_{+}$.

Proposition 2.6. Let $S$ be a uniformly bounded $\sigma(\mathscr{Z}, \mathscr{F})$-continuous semigroup which is locally $\tau(\mathscr{X}, \mathscr{F})$-equicontinuous. Further let $\mathscr{N}$ be a norm bounded set of finite measures on $\mathbf{R}_{+}$such that

$$
\lim _{r \rightarrow \infty} \int_{r}^{\infty} d|\mu|(t)=0
$$

uniformly for $\mu \in \mathscr{N}$.

It follows that the family $\left\{S_{\mu} ; \mu \in \mathscr{N}\right\}$ is $\tau(\mathscr{X}, \mathscr{F})$-equicontinuous.

Proof. It follows from the assumption $(*)$ that there exists a continuous, positive, decreasing function $\rho$ on $\mathbf{R}_{+}$which tends to zero at infinity such that

$$
B=\sup \left\{\int_{0}^{\infty} d|\mu|(t) / \rho(t) ; \mu \in \mathscr{N}\right\}<+\infty .
$$

Let $G_{t}=\rho(t) S_{t}$ if $0 \leq t<\infty$ and $G_{t}=0$ if $t=\infty$. Then $(t, f) \mapsto G_{t}^{*} f$ is jointly continuous on bounded subsets of $\mathscr{F}$ for $t \in[0, \infty]$. (It is continuous for finite $t$ by Proposition 2.4 and continuous at infinity because $\left\|G_{t}\right\| \rightarrow 0$ as $t \rightarrow \infty$.) Hence $\left\{G_{t} ; t \in[0, \infty]\right\}$ is equicontinuous by Remark 2.5. Thus for any $\sigma(\mathscr{F}, \mathscr{X})$-compact set $K^{\prime}$ such that $p_{K}\left(\rho(t) S_{t} x\right) \leq p_{K}^{\prime}(x)$ for all $t \in[0, \infty)$ and all $x \in \mathscr{X}$. Therefore

$$
p_{K}\left(S_{\mu} x\right) \leq \int d|\mu|(t) \rho(t)^{-1} p_{K}\left(\rho(t) S_{t} x\right) \leq B p_{K^{\prime}}(x)
$$

for all $\mu \in N$ and $x \in \mathscr{Z}$, as desired. 
CoROLlary 2.7. Let $S$ be a uniformly bounded $\sigma(\mathscr{Z}, \mathscr{F})$-continuous semigroup which is locally $\tau(\mathscr{X}, \mathscr{F})$-equicontinuous. If $\mu$ is a finite measure on $\mathbf{R}_{+}$ then $S_{\mu}$ is $\tau(\mathscr{X}, \mathscr{F})$-continuous.

Proof. If the set $\mathscr{N}$ in Proposition 2.6 is finite the uniformity condition $(*)$ is irrelevant and the family $\left\{S_{\mu} ; \mu \in \mathscr{N}\right\}$ is equicontinuous.

We conclude this section with some remarks about open questions concerning the foregoing framework.

First, it is conceivable that local equicontinuity of a $\sigma(\mathscr{X}, \mathscr{F})$-continuous semigroup follows automatically from the assumed properties of $\mathscr{F}$. We have argued, in the discussion prior to Proposition 2.4, that this is the case if $\mathscr{F}=\mathscr{Z}^{*}$ or if $\mathscr{F}=\mathscr{X}_{*}$. But equicontinuity is not automatic. Translations on $L_{\infty}(\mathbf{R})$ are locally equicontinuous but not equicontinuous with respect to the $\sigma\left(L_{\infty}, L_{1}\right)$-topology, that is, the weak*-topology. Nevertheless the dual group, translations on $L_{1}(R)$, is equicontinuous.

Second, if local equicontinuity is not automatic it is still possible that it is a self-dual property, that is the $\sigma(\mathscr{X}, \mathscr{F})$-continuous semigroup $S=\left\{S_{t}\right\}_{t \geq 0}$ is locally equicontinuous if, and only if, the dual semigroup $S^{*}=\left\{S_{t}^{*}\right\}_{t \geq 0}$ is locally equicontinuous. Right and left translations on $L_{1}\left(\mathbf{R}_{+}\right)$and $L_{\infty}\left(\mathbf{R}_{+}\right)$ show that equicontinuity of a semigroup does not imply equicontinuity of the dual semigroup but the situation for local equicontinuity is unclear.

(The properties of $L_{1}(\mathbf{R})$ and $L_{\infty}(\mathbf{R})$ alluded to above rely upon an appropriate characterization of weakly compact sets in $L_{1}$. The required characterization follows from Theorem V.6.1 and Exercise IV.13.54 of [8]. The solution of the exercise follows easily from Theorem IV.8.9 and Corollary IV.8.11.)

\section{Distributions and semigroups}

Throughout this section the $\sigma(\mathscr{X}, \mathscr{F})$-continuous semigroup $S=\left\{S_{t}\right\}_{t \geq 0}$ is assumed to be uniformly bounded, that is, $\left\|S_{t}\right\| \leq M$ for some $M \geq 1$ and all $t \geq 0$. In this case one can associate with each bounded measure $\mu$ on $\mathbf{R}_{+}$ the bounded $\sigma(\mathscr{X}, \mathscr{F})$-continuous operator $S_{\mu}$ by

$$
S_{\mu} x=\int d \mu(t) S_{t} x
$$

Now, following Schwartz [18], we extend this definition to a large class of distributions supported on $\mathbf{R}_{+}$.

Let $\varphi$ denote a tempered distribution with support in $\mathbf{R}_{+}$. Then $\varphi$ is defined to be $\mathbf{R}_{+}$-summable if $\varphi * \eta \in L_{1}\left(\mathbf{R}_{+}\right)$for each $\eta \in C_{00}^{\infty}\left(\mathbf{R}_{+}\right)$, the infinitely 
often-differentiable functions with compact support in $(0, \infty)$, where $\varphi * \eta$ denotes the usual convolution product. Thus if $\varphi$ is $\mathbf{R}_{+}$-summable one can define the bounded operators $S_{\varphi * \eta}$ by

$$
S_{\varphi * \eta} x=\int_{0}^{\infty} d t(\varphi * \eta)(t) S_{t} x, \quad x \in \mathscr{X} .
$$

Next let $F$ denote the filter with base formed by the $\eta_{\varepsilon} \in C_{00}^{\infty}\left(\mathbf{R}_{+}\right)$such that the support of $\eta_{\varepsilon}$ is contained in $(0, \varepsilon), \eta_{\varepsilon} \geq 0$, and $1-\varepsilon \leq \int d t \eta_{\varepsilon}(t) \leq 1+\varepsilon$. Then we define $S_{\varphi}$ by

$$
D\left(S_{\varphi}\right)=\left\{x ; \sigma(\mathscr{X}, \mathscr{F})-\lim S_{\varphi * \eta} x \text { exists }\right\}
$$

and

$$
S_{\varphi} x=\sigma(\mathscr{X}, \mathscr{F})-\lim S_{\varphi * \eta} x
$$

for $x \in D\left(S_{\varphi}\right)$.

Alternately if $\eta \in C_{00}^{\infty}\left(\mathbf{R}_{+}\right), \eta \geq 0, \int d t \eta(t)=1$, and one sets $\eta_{n}(t)=n \eta(n t)$ then one can define an operator $S_{\varphi}^{\eta}$ by a similar process;

$$
D\left(S_{\varphi}^{\eta}\right)=\left\{x ; \sigma(\mathscr{Z}, \mathscr{F})-\lim S_{\varphi * \eta_{n}} x \text { exists }\right\}
$$

and

$$
S_{\varphi}^{n} x=\sigma(\mathscr{Z}, \mathscr{F})-\lim S_{\varphi * \eta_{n}} x
$$

for $x \in D\left(S_{\varphi}^{n}\right)$. Clearly $S_{\varphi} \subseteq S_{\varphi}^{\eta}$ but in fact the two operators are equal. This is part of the following basic result.

Proposition 3.1. Let $S$ be a uniformly bounded $\sigma(\mathscr{X}, \mathscr{F})$-continuous semigroup and $\varphi$ on $\mathbf{R}_{+}$-summable distribution. Then

1. $S_{\varphi}$ is a $\sigma(\mathscr{X}, \mathscr{F})-\sigma(\mathscr{X}, \mathscr{F})$-closed operator,

2. $\mathscr{X}_{\text {reg }}(S)$ is a $\sigma(\mathscr{Z}, \mathscr{F})$-dense core of $S_{\varphi}$,

3. $S_{\varphi}=S_{\varphi}^{\eta}$.

Proof. 1. Assume $x_{j} \in D\left(S_{\varphi}\right)$, and $x_{j} \rightarrow x, S_{\varphi} x_{j} \rightarrow y$ in the $\sigma(\mathscr{X}, \mathscr{F})$ topology. Then for $\eta \in F$ one has $S_{\eta} x_{j} \in D\left(S_{\varphi}\right)$ and $S_{\varphi} S_{\eta} x_{j}=S_{\varphi * \eta} x_{j} \rightarrow$ $S_{\varphi * \eta} x$ because $S_{\varphi * \eta}$ is a bounded $\sigma(\mathscr{X}, \mathscr{F})$-continuous operator. But $S_{\varphi} S_{\eta} x_{j}$ $=S_{\eta} S_{\varphi} x_{j} \rightarrow S_{\eta} y$. Therefore $S_{\varphi * \eta} x=S_{\eta} y$. Finally

$$
\sigma(\mathscr{X}, \mathscr{F})-\lim S_{\eta} y=y
$$

and one concludes that $x \in D\left(S_{\varphi}\right)$ and $S_{\varphi} x=y$.

2. The proof is identical to the proof of part 2 of Proposition 2.1. 
3. Clearly $S_{\varphi} \subseteq S_{\varphi}^{\eta}$. But if $x \in D\left(S_{\varphi}^{\eta}\right)$ and $\xi \in F$ then

$$
\begin{aligned}
\sigma(\mathscr{X}, \mathscr{F})-\lim S_{\varphi * \xi} S_{\eta_{n}} x & =\sigma(\mathscr{X}, \mathscr{F})-\lim S_{\varphi * \xi * \eta_{n}} x \\
& =S_{\varphi * \eta_{n}} x .
\end{aligned}
$$

Thus $S_{\eta_{n}} x \in D\left(S_{\varphi}\right)$. But

$$
\sigma(\mathscr{X}, \mathscr{F})-\lim S_{\eta_{n}} x=x
$$

and

$$
\sigma(\mathscr{X}, \mathscr{F})-\lim S_{\varphi} S_{\eta_{n}} x=\sigma(\mathscr{X}, \mathscr{F})-\lim S_{\varphi * \eta_{n}} x=S_{\varphi}^{\eta} x .
$$

Since $S_{\varphi}$ is $\sigma(\mathscr{Z}, \mathscr{F})-\sigma(\mathscr{X}, \mathscr{F})$-closed this establishes that $x \in D\left(S_{\varphi}\right)$ and $S_{\varphi} x=S_{\varphi}^{\eta} x$. Thus $S_{\varphi}=S_{\varphi}^{\eta}$.

Next we consider products of the $S_{\varphi}$.

Proposition 3.2. Let $S$ be a uniformly bounded $\sigma(\mathscr{Z}, \mathscr{F})$-continuous semigroup and $\varphi_{1}, \varphi_{2}$ two $\mathbf{R}_{+}$-summable distributions such that $\varphi_{1} * \varphi_{2}$ is $\mathbf{R}_{+}^{-}$ summable. Then

$$
S_{\varphi_{1}} S_{\varphi_{2}} \subseteq S_{\varphi_{1} * \varphi_{2}}
$$

but if $D\left(S_{\varphi_{2}}\right) \supseteq D\left(S_{\varphi_{1} * \varphi_{2}}\right)$ then

$$
S_{\varphi_{1}} S_{\varphi_{2}}=S_{\varphi_{1} * \varphi_{2}} .
$$

In particular one has equality whenever $S_{\varphi_{2}}$ is bounded.

Proof. Let $\eta_{1}, \eta_{2} \in F$ then

$$
S_{\varphi_{1} * \eta_{1}} S_{\varphi_{2} * \eta_{2}} x=S_{\left(\varphi_{1} * \eta_{1}\right) *\left(\varphi_{2} * \eta_{2}\right)} x=S_{\left(\varphi_{1} * \varphi_{2}\right) *\left(\eta_{1} * \eta_{2}\right)} x
$$

for all $x \in \mathscr{Z}$. Thus if $x \in D\left(S_{\varphi_{2}}\right)$ then

$$
S_{\varphi_{1} * \eta_{1}} S_{\varphi_{2}} x=S_{\left(\varphi_{1} * \varphi_{2}\right) * \eta_{1}} x .
$$

Hence $x \in D\left(S_{\varphi_{1} * \varphi_{2}}\right)$ if, and only if, $S_{\varphi_{2}} x \in D\left(S_{\varphi_{1}}\right)$ and then

$$
S_{\varphi_{1}} S_{\varphi_{2}} x=S_{\varphi_{1} * \varphi_{2}} x \text {. }
$$

This establishes that $S_{\varphi_{1} * \varphi_{2}}$ extends $S_{\varphi_{1}} S_{\varphi_{2}}$. It also establishes that if $D\left(S_{\varphi_{1} * \varphi_{2}}\right)$ $\subseteq D\left(S_{\varphi_{2}}\right)$ then $D\left(S_{\varphi_{1} * \varphi_{2}}\right) \subseteq D\left(\varphi_{1} S_{\varphi_{2}}\right)$. Thus $S_{\varphi_{1} * \varphi_{2}}=S_{\varphi_{1}} S_{\varphi_{2}}$.

Next we consider continuity properties of the map $\varphi \mapsto S_{\varphi}$. These properties are more delicate because the $S_{\varphi}$ are generally unbounded. Nevertheless $\mathscr{X}_{\text {reg }}(S)$ is a common core for all the $S_{\varphi}$ and one has the following weak result. 
Proposition 3.3. Let $S$ be a uniformly bounded $\sigma(\mathscr{X}, \mathscr{F})$-continuous semigroup and $\varphi_{j}, \varphi, \mathbf{R}_{+}$-summable distributions such that $\varphi_{\eta}$ is $L_{1}$-convergent to $\varphi * \eta$ for each $\eta \in C_{00}^{\infty}\left(\mathbf{R}_{+}\right)$. If $S_{\varphi}^{0}$ is defined by

$$
D\left(S_{\varphi}^{0}\right)=\left\{x ; \sigma(\mathscr{X}, \underset{j}{F})-\lim S_{\varphi_{j}} \text { exists }\right\}
$$

and

$$
S_{\varphi}^{0} x=\sigma(\mathscr{Z}, \mathscr{F})-\lim S_{\varphi_{j}} x
$$

for $x \in D\left(S_{\varphi}^{0}\right)$ then $S_{\varphi}^{0}$ is $\sigma(\mathscr{X}, \mathscr{F})-\sigma(\mathscr{X}, \mathscr{F})$-closable and its closure $S_{\varphi}^{0}$ equals $S_{\varphi}$.

Proof. If $x \in D\left(S_{\varphi}^{0}\right)$ and $\eta \in C_{00}^{\infty}\left(\mathbf{R}_{+}\right)$then

$$
S_{\eta} S_{\varphi}^{0} x=\sigma(\mathscr{X}, \mathscr{F})-\lim S_{\eta} S_{\varphi} x=\sigma(\mathscr{X}, \mathscr{F})-\lim S_{\varphi_{\eta}} x=S_{\varphi * \eta} x=S_{\varphi} S_{\eta} x .
$$

Here we have used the fact that $L_{1}$-convergence of $\varphi_{\eta}$ to $\varphi * \eta$ implies $\sigma(\mathscr{Z}, \mathscr{F})$-convergence of $S_{\varphi, * \eta} x$ to $S_{\varphi * \eta} x$. But now one deduces that

$$
\sigma(\mathscr{X}, \mathscr{F})-\lim S_{\varphi} S_{\eta} x=S_{\varphi}^{0} x
$$

Since, however,

$$
\sigma(\mathscr{Z}, \mathscr{F})-\lim S_{\eta} x=x
$$

and $S_{\varphi}$ is closed this establishes that $S_{\varphi}^{0} \subseteq S_{\varphi}$. In particular, $S_{\varphi}^{0}$ is closable. But $\mathscr{X}_{\text {reg }}(S) \subseteq D\left(S_{\varphi}^{0}\right)$ and $\mathscr{X}_{\text {reg }}(S)$ is a core of $S_{\varphi}$. Therefore $\overline{S_{\varphi}^{0}}=S_{\varphi}$.

Remark 3.4. It suffices for Proposition 3.3 that $\varphi_{j} * \eta$ is $L_{1}$-convergent to $\varphi * \eta$ for one non-zero $\eta \in C_{00}^{\infty}\left(\mathbf{R}_{+}\right)$. The proof is very similar but one takes a limit in the manner used to prove the last part of Proposition 3.1.

Remark 3.5. The weakness of Proposition 3.3 is that $S_{\varphi}^{0}$ is not necessarily closed. If it is known for other reasons to be closed then of course $S_{\varphi}^{0}=S_{\varphi}$.

Finally we note that the foregoing results are essentially due to Schwartz [18]. But Schwartz considers the class of distributions which are finite sums of derivatives of finite measures. This class has the advantage of being automatically closed under convolution.

\section{Fourier transforms}

In this section we establish our notation for Fourier transforms and prove a number of results about transforms of particular distributions. 
If $\eta \in \mathscr{S}(\mathbf{R})$ we define its Fourier transform $\tilde{\eta}$ by

$$
\tilde{\eta}(\lambda)=(2 \pi)^{-1} \int_{-\infty}^{\infty} d t e^{-i \lambda t} \eta(t)
$$

and if $\varphi$ is a tempered distribution we define its Fourier transform $\hat{\varphi}$ by the formal relation

$$
\left.\left.\int d \operatorname{t\eta }(t) \varphi(t)=\int d \lambda \tilde{\eta}(\lambda) \hat{\varphi}\right) \lambda\right)
$$

or, even more formally, by

$$
\hat{\varphi}(\lambda)=\int_{-\infty}^{\infty} d t e^{i \lambda t} \varphi(t)
$$

With these conventions the Fourier transform of a distribution $\varphi$ with support in the positive half-line $[0, \infty)$ is analytic in the upper half-plane, and the Fourier transform of the convolution product of two tempered distributionswhere defined-is the product of the Fourier transforms, without any extra factors of $2 \pi$.

Next for any positive integer $n$ the $n$th derivative of the Dirac measure $\delta$, which will be denote by $\delta^{n}$, has Fourier transform $\lambda \mapsto(-i \lambda)^{n}$. More generally if $\alpha$ is any real number larger than -1 we define the distribution $\delta^{\alpha}$ to be the inverse Fourier transform of $\lambda \mapsto(-i \lambda)^{\alpha}$. Here, and in the sequel, the branch of a fractional power is defined as follows. If $z$ is a nonzero complex number then $z^{\alpha}$ means $\exp \{\alpha \log z\}$ where the imaginary part of $\log z$ is chosen to be in $(-\pi, \pi]$. Thus the logarithm, and non-integral powers, are cut along the negative real axis, and $(-i z)^{\alpha}$ is continuous on the closed upper half-plane.

The following result is well known.

Proposition 4.1. The distribution $\delta^{\alpha}$ has support in $[0, \infty)$ and on $(0, \infty)$ it is equal to

$$
t \rightarrow-\frac{\operatorname{Sin} \pi \alpha}{\pi} \Gamma(\alpha+1) \frac{1}{t^{\alpha+1}}
$$

Proof. By the definition of $\delta^{\alpha}, \int d t \eta(t) \delta^{\alpha}(t)=\int d \lambda \tilde{\eta}(\lambda)(-\lambda)^{\alpha}$ and our choice of $z^{\alpha}$ makes $(-\lambda)^{\alpha}$ analytic in the upper half-plane. If $\eta$ has support in $(-\infty, 0)$ then $\tilde{\eta}$ is also analytic in the upper half-plane and a straightforward application of the Cauchy Integral Theorem gives $\int d \lambda \tilde{\eta}(\lambda)(i \lambda)^{\alpha}=0$. Hence the support of $\delta^{\alpha}$ is contained in $[0, \infty)$. 
If, on the other hand, $\eta$ has support in $(0, \infty)$, we can deform the contour of integration to the negative imaginary axis; then

$$
\begin{aligned}
\int_{-\infty}^{\infty} d \lambda \tilde{\eta}(\lambda)(-i \lambda)^{\alpha} & =(-i) \int_{0}^{\infty} d \mu \tilde{\eta}(-i \mu) \mu^{\alpha}\left(e^{-i \pi \alpha}-e^{i \pi \alpha}\right) \\
& =-2 \operatorname{Sin} \pi \alpha \int_{0}^{\infty} d \mu \tilde{\eta}(-i \mu) \mu^{\alpha} \\
& =\frac{-\operatorname{Sin} \pi \alpha}{\pi} \int_{0}^{\infty} d \mu \mu^{\alpha} \int_{0}^{\infty} d t e^{-\mu t} \eta(t) \\
& =\frac{-\operatorname{Sin} \pi \alpha}{\pi} \int_{0}^{\infty} d t \eta(t) t^{-\alpha-1} \int_{0}^{\infty} d \mu \mu^{\alpha} e^{-\mu} \\
& =\frac{-\sin \pi \alpha}{\pi} \Gamma(\alpha+1) \int_{0}^{\infty} d t \eta(t) t^{-\alpha-1}
\end{aligned}
$$

as desired.

Corollary 4.2. 1. For $\alpha>0$, the distribution $\delta^{\alpha}$ can be written as the sum of a distribution of compact support and an integrable function,

2. If $\eta \in S(\mathbf{R})$ then $\left(\eta * \delta^{\alpha}\right)(t)=O\left(|t|^{1-\alpha}\right)$ for large $t$. In particular $\eta * \delta^{\alpha} \in L^{1}(\mathbf{R})$ for $\alpha>0$.

Proof. Let $\xi$ be a $C^{\infty}$-function of compact support equal to 1 in a neighbourhood of the origin. Then $\xi \delta^{\alpha}$ is a distribution of compact support and $(1-\xi) \delta^{\alpha}$ is an infinitely-differentiable function with support in $(0, \infty)$ and equal to a constant multiple of $t^{-1-\alpha}$ for sufficiently large $t$. IF $\eta \in S(\mathbf{R})$ then $\eta * \xi \delta^{\alpha} \in \mathscr{S}(\mathbf{R})$ and

$$
\left(\eta *(1-\xi) \delta^{\alpha}\right)(t)=O\left(|t|^{-1-\alpha}\right) .
$$

In the rest of the section our aim is to prove the integrability of the inverse Fourier transforms of some special simple functions. We begin with two essentially standard results of this nature.

Proposition 4.3. Let $\alpha>0$. Then the Fourier transform of the distribution $t \mapsto \theta(t) t^{\alpha-1} e^{-t}$, where $\theta(t)=0$ for $t<0$ and $\theta(t)=1$ for $t>0$, is $\Gamma(\alpha)(1-i \lambda)^{-\alpha}$. In particular $(1-i \lambda)^{-\alpha}$ is the Fourier transform of an integrable function.

Proof. For any $\eta \in \mathscr{S}(\mathbf{R})$

$$
\begin{aligned}
\int_{0}^{\infty} d t t^{\alpha-1} e^{-t} \eta(t) & =\int_{0}^{\infty} d t t^{\alpha-1} e^{-t} \int_{-\infty}^{\infty} d \lambda e^{i \lambda t} \tilde{\eta}(\lambda) \\
& =\int_{-\infty}^{\infty} d \lambda \tilde{\eta}(\lambda) \int_{0}^{\infty} d t t^{\alpha-1} e^{-(1-i \lambda) t}
\end{aligned}
$$


Now changing variables in the second integral from $t$ to $s=(1-i \lambda) t$ and using the Cauchy Integral Theorem to move the contour of integration back to the positive real axis one finds

$$
\int_{0}^{\infty} d t t^{\alpha-1} e^{-(1-i \lambda) t}=(1-i \lambda)^{-\alpha} \int_{0}^{\infty} d s s^{\alpha-1} e^{-s}=\Gamma(\alpha)(1-i \lambda)^{-\alpha} .
$$

Proposition 4.4. If $\alpha>0$ then $\lambda \mapsto(-i \lambda /(1-i \lambda))^{\alpha}-1$ is the Fourier transform of an integrable function.

Proof. One has $(-i \lambda /(1-i \lambda))^{\alpha}-1=(1-1 /(1-i \lambda))^{\alpha}-1=\alpha /(1-i \lambda)+$ $R(\lambda)$. But the first term is the Fourier transform of an integrable function by Proposition 4.3 and the remainder term by a straightforward argument. (The remainder and its derivatives are of order $\lambda^{-2}$ at infinity.)

The last integrability result is the most delicate. It is the basis of our discussion of fractional derivatives in Section 6.

Proposition 4.5. If $\alpha>0$ then $\lambda \mapsto\left(\left(1-e^{i \lambda}\right) /(-i \lambda)\right)^{\alpha}$ is the Fourier transform of an integrable function.

Proof. The proof is divided into three cases.

Case 1. $\alpha=1$. Then $\lambda \rightarrow\left(1-e^{i \lambda}\right) /(-i \lambda)$ is the Fourier transform of the characteristic function of the interval $(0,1)$.

Case 2. $\alpha>1$. Define $\hat{g}_{\alpha}$ by $\hat{g}_{\alpha}(\lambda)=\left(\left(1-e^{i \lambda}\right) /(-i \lambda)\right)^{\alpha}$. Then $\hat{g}_{\alpha}$ is integrable and hence its inverse Fourier transform $g_{\alpha}$ is a continuous function. Moreover $\hat{g}_{\alpha}$ is continuously differentiable, and its derivative $\hat{g}_{\alpha}^{\prime}$ is integrable, and it is twice continuously except at the points $2 \pi j, j= \pm 1, \pm 2, \ldots$, where the second derivative $\hat{g}_{\alpha}^{\prime \prime}$ has integrable singularities; $\hat{g}_{\alpha}^{\prime \prime}$ is globally integrable. It follows readily that the pointwise second derivative of $\hat{g}_{\alpha}$, defined almost everywhere, coincides with its second derivative in the sense of distributions. Since this second derivative is an integrable function, $g_{\alpha}(t)=0\left(t^{2}\right)$ at infinity and hence $g_{\alpha}$ is integrable.

Case 3. $0<\alpha<1$. It follows from the binomial theorem that $\left(1-e^{i \lambda}\right)^{\alpha}=$ $\sum_{j=0}^{\infty} c_{j} e^{i \lambda j}$ where $\left|c_{j}\right|=O\left(j^{-1-\alpha}\right)$. Since the $c_{j}$ are absolutely summable, the series

$$
\left(\left(1-e^{i \lambda}\right) /(-i \lambda)\right)^{\alpha}=\sum_{j=0}^{\infty} c_{j} e^{i \lambda j} /(-i \lambda)^{\alpha}
$$

converges in the sense of distributions. By Proposition 4.1, $\lambda \mapsto(-i \lambda)^{-\alpha}$ is a constant multiple of the Fourier transform of $\theta(t) t^{\alpha-1}$. Absorbing the 
constant factor into the $c_{j}$ we obtain the representation

$$
g_{\alpha}(t)=\sum_{j=0}^{\infty} c_{j} \theta(t-j)(t-j)^{\alpha-1} .
$$

For any fixed $t$ there are only finitely many non-zero terms in this sum so $g_{\alpha}$ is certainly a locally integrable function. We have to show that it is globally integrable, that is, that the series

$$
k \mapsto \int_{k}^{k+1} d t\left|g_{\alpha}(t)\right|
$$

is summable.

We start by noting that $g_{\alpha+1}=g_{\alpha} * g_{1}$, that is,

$$
g_{\alpha+1}(t)=\int_{0}^{1} d s g_{\alpha}(t-s)=\int_{t-1}^{t} d s g_{\alpha}(s)
$$

Thus by Case 2 above

$$
\int_{k}^{k+1} d t g_{\alpha}(t)=O\left(k^{-2}\right)
$$

In order to deduce from this that the series $(*)$ is summable we are going to argue that $g_{\alpha}$ does not vary too much on $(k, k+1)$ for large $k$. From the above representation of $g_{\alpha}$ we first find for $k<t<k+1$ that

$$
G_{\alpha}(t)=\sum_{j=0}^{k} c_{j}(t-j)^{\alpha-1}
$$

We will estimate the term with $j=k$ and the remainder separately. Thus we write $h(t)=\sum_{j=0}^{k-1} c_{j}(t-j)^{\alpha-1}$. Then $h^{\prime}(t)=(\alpha-1) \sum_{j=0}^{k-1} c_{j}(t-j)^{\alpha-2}$ and so $\left|h^{\prime}(t)\right| \leq(1-\alpha) \sum_{j=0}^{k-1}\left|c_{j}\right|(k-j)^{\alpha-2}$ for $k<t<k+1$. Let $k_{1}$ denote the integer part of $k / 2$, and split the sum into a sum from 0 to $k_{1}$ and a sum from $k_{1}+1$ to $k-1$; then

$$
\sum_{j=0}^{k_{1}}\left|c_{j}\right|(k-j)^{\alpha-2} \leq(2 / k)^{2-\alpha} \sum_{j=0}^{k_{1}}\left|c_{j}\right| \leq \text { constant } \times k^{\alpha-2},
$$

and

$$
\begin{aligned}
\sum_{j=k_{1}+1}^{k-1}\left|c_{j}\right|(k-j)^{\alpha-2} & \leq \text { constant } \times k^{1-\alpha} \sum_{j=k_{1}+1}^{k-1}(k-j)^{\alpha-2} \\
& \leq \text { constant } \times k^{-1-\alpha}
\end{aligned}
$$


Thus $\left|h^{\prime}(t)\right| \leq c k^{1-\beta}$ where $\beta$ is the smaller of $\alpha$ and $1-\alpha$ and $c$ is independent of $k$. Now

$$
\begin{aligned}
\left|g_{\alpha}(t)-h\left(k+\frac{1}{2}\right)\right| & \leq\left|g_{\alpha}(t)-h(t)\right|+\left|h(t)-h\left(k+\frac{1}{2}\right)\right| \\
& \leq\left|c_{k}\right|(t-k)^{\alpha-1}+c k^{-1-\beta}
\end{aligned}
$$

for $k<t<k+1$. Hence

$$
\left|\int_{k}^{k+1} d t g_{\alpha}(t)-h\left(k+\frac{1}{2}\right)\right|=O\left(k^{-1-\beta}\right) .
$$

Since, however, $\int_{k}^{k+1} d t g_{\alpha}(t)=O\left(k^{-2}\right)$ one finds $\left|h\left(k+\frac{1}{2}\right)\right|=O\left(k^{-1-\beta}\right)$. Thus writing

$$
\left|g_{\alpha}(t)\right| \leq\left|h\left(k+\frac{1}{2}\right)\right|+\left|c_{k}\right|(t-k)^{\alpha-1}+c k^{-1-\beta}
$$

one obtains $\int_{k}^{k+1} d t\left|g_{\alpha}(t)\right|=O\left(k^{-1-\beta}\right)$ as desired.

\section{Fractional powers}

Throughout this section $S$ denotes a uniformly bounded locally equicontinuous $\sigma(\mathscr{X}, \mathscr{F})$-continuous semigroup with generator $H$. For $\alpha>0$ we define the fractional power $H^{\alpha}$ of $H$ to be equal to the operator $S_{\delta^{\alpha}}$. Thus $H^{\alpha}$ is a $\sigma(\mathscr{X}, \mathscr{F})-\sigma(\mathscr{X}, \mathscr{F})$-closed operator and $\mathscr{X}_{\text {reg }}(S)$ is a $\sigma(\mathscr{X}, \mathscr{F})$-dense core of $H^{\alpha}$, by Proposition 3.1. Our aim is to first prove that $H^{\alpha}$ defined in this way has the elementary properties of a fractional power, for example $H^{\alpha} H^{\beta}=H^{\alpha+\beta}$, and second to establish that $H^{\alpha}$ coincides with the fractional power of $H$ as defined by other means.

We begin by noting that $I+H$ generates the semigroup $S^{e}=\left\{e^{-t} S_{t}\right\}_{t \geq 0}$ which has boundedness and continuity properties similar to those of $S$. Hence we can define $(I+H)^{\alpha}$ as the operator $S_{\delta^{\alpha}}^{e}$. Alternatively if we denote the tempered distribution $t \mapsto e^{-t} \delta^{\alpha}(t)$ by $\gamma_{\alpha}$ then

$$
(I+H)^{\alpha}=S_{\gamma_{\alpha}} .
$$

Now by the definition of $\delta^{\alpha}$, and a standard fact about Fourier transforms, the Fourier transform of $\gamma_{\alpha}$ is $(1-i \lambda)^{\alpha}$. Next we use $r_{\alpha}$ to denote the inverse Fourier transform of $(1-i \lambda)^{-\alpha}$. Then by Proposition $4.3, r_{\alpha}(t)$ is a constant multiple of $t^{\alpha-1} e^{-t}$, and in particular it is an integrable function. Thus defining $R_{\alpha}=S_{r_{\alpha}}$ we conclude that $R_{\alpha}$ is a bounded $\sigma(\mathscr{X}, \mathscr{F})$-continuous operator on $\mathscr{X}$.

THEOREM 5.1 (a) $(I+H)^{\alpha}=R_{\alpha}^{-1}$ in the strict algebraic sense, that is, $R_{\alpha}$ and $(I+H)^{\alpha}$ both have kernel $\{0\}$ and $D\left((I+H)^{\alpha}\right)=R\left(R_{\alpha}\right)$, the range of $R_{\alpha}$.

(b) $D\left(H^{\alpha}\right)=D\left((I+H)^{\alpha}\right)$. 
Proof. (a) One has $\gamma_{\alpha} * r_{\alpha}=\delta$ and hence $R_{\alpha}(I+H)^{\alpha} x=x$, for all $x \in D\left((I+H)^{\alpha}\right)$, and $(I+H)^{\alpha} R_{\alpha} x=x$, for all $x \in X$, by Proposition 3.2. This implies that both $R_{\alpha}$ and $(I+H)^{\alpha}$ have kernel $\{0\}$ and $\left(I+H_{\alpha}\right) \supseteq R_{\alpha}^{-1}$. But the range of $R_{\alpha}^{-1}$ is all of $\mathscr{Z}$ so any proper extension of $R_{\alpha}^{-1}$ must have nontrivial kernel. Since $(I+H)^{\alpha}$ has trivial kernel one concludes that $(I+H)^{\alpha}=R_{\alpha}^{-1}$.

(b) Let $\mu$ denote the inverse Fourier transform of $(-i \lambda /(1-i \lambda))^{\alpha}$. Then $\mu$ is a finite measure by Proposition 4.4. Since $\mu * \gamma_{\alpha}=\delta^{\alpha}$ it follows from Proposition 3.2 that $S_{\mu}(I+H)^{\alpha} x=H^{\alpha} x$ for all $x \in D\left((I+H)^{\alpha}\right)$. In particular $D\left((I+H)^{\alpha}\right) \subseteq D(H)^{\alpha}$. To obtain the opposite inclusion we use the following lemma.

Lemma 5.2. There exist finite measures $r_{1}$ and $r_{2}$ such that

$$
(1-i \lambda)^{\alpha}=\hat{r}_{1}(\lambda)(-i \lambda)^{\alpha}+\hat{r}_{2}(\lambda)
$$

Proof. Using the binomial theorem we write

$$
\begin{aligned}
((1-i \lambda) /(-i \lambda))^{\alpha} & =\left(1-(1-i \lambda)^{-1}\right)^{-\alpha} \\
& =1=\sum_{j=1}^{n} c_{j}^{\alpha}(1-i \lambda)^{-j}+O\left(|\lambda|^{-n-1}\right)
\end{aligned}
$$

where $n$ is any integer such that $n>\alpha$. We then take

$$
\hat{r}_{1}(\lambda)=1+\sum_{j=1}^{n} c_{j}^{\alpha}(1-i \lambda)^{-j}
$$

and

$$
\hat{r}_{2}(\lambda)=(1-i \lambda)^{\alpha}-\hat{r}_{1}(\lambda)(-i \lambda)^{\alpha}=(-i \lambda)^{\alpha}\left[((1-i \lambda) /(i \lambda))^{\alpha}-\hat{r}_{1}(\lambda)\right] .
$$

Then $\hat{r}_{1}(\lambda)$ is the Fourier transform of a finite measure by Proposition 4.3. But from the above expression $\hat{r}_{2}(\lambda)=O\left(|\lambda|^{-n-1+\alpha}\right)$ for large $\lambda$ and hence it is integrable at infinity as are all its derivatives. It is also analytic everywhere on the real axis except at the origin, where it can be written locally as the sum of two terms the first of which is analytic and the second of which is a product of an analytic function by $(-i \lambda)^{\alpha}$. Hence by Corollary $4.2 .2 \hat{r}_{2}(\lambda)$ is the Fourier transform of a finite measure.

Now we return to the proof of Theorem 5.1.

Using Lemma 5.2 we have $\gamma_{\alpha}-r_{2}=r_{1} * \delta^{\alpha}$ and again by Proposition 3.2 $D\left(S_{r_{1} * \delta^{\alpha}}\right) \supseteq D\left(S_{\delta^{\alpha}}\right)=D\left(H^{\alpha}\right)$. But on the other hand $D\left(S_{\gamma_{a}-r_{2}}\right)=D\left(S_{\gamma_{n}}\right)=$ $D\left((I+H)^{\alpha}\right)$. Therefore $D\left((I+H)^{\alpha} \supseteq D\left(H^{\alpha}\right)\right.$ which combined with the earlier conclusion gives equality of the two domains.

Next we prove that the $H^{\alpha}$ have the basic properties of fractional powers. 
TheOREM 5.3. a. If $\alpha, \beta \geq 0$ then $H^{\alpha} H^{\beta}=H^{\alpha+\beta}$.

b. If $\alpha=n$ is a positive integer than $H^{\alpha}$ is equal to the $n$-fold product of $H$.

Proof. a. Since $\delta^{\alpha} * \delta^{\beta}=\delta^{\alpha+\beta}$ it suffices by Proposition 3.2 to prove that $D\left(H^{\alpha+\beta}\right) \subseteq D\left(H^{\beta}\right)$. But $D\left(H^{\alpha+\beta}\right)$ is equal to the range of $R_{\alpha+\beta}$ by Theorem 5.1. Moreover since $r_{\alpha+\beta}=r_{\alpha} * r_{\beta}$ it follows that $R_{\alpha+\beta}=R_{\beta} R_{\alpha}$, by another application of Proposition 3.2, and hence the range of $R_{\beta}$ contains the range of $R_{\alpha+\beta}$. Thus $D\left(H^{\beta}\right) \supseteq D\left(H^{\alpha+\beta}\right)$.

b. It follows from Proposition 5.1 that $S_{\delta^{\prime}}$ is a $\sigma(\mathscr{X}, \mathscr{F})-\sigma(\mathscr{X}, \mathscr{F})$-closed operator and $\mathscr{X}_{\text {reg }}(S)$ is a $\sigma(\mathscr{X}, \mathscr{F})$-dense core. Similarly by Proposition 2.1 $H$ is a $\sigma(\mathscr{X}, \mathscr{F})-\sigma(\mathscr{X}, \mathscr{F})$-closed operator and $\mathscr{X}_{\text {reg }}(S)$ is a $\sigma(\mathscr{X}, \mathscr{F})$-dense core. But if $x=S_{\eta} y \in \mathscr{X}_{\text {reg }}(S)$ then

$$
S_{\delta^{\prime}} S_{\eta} y=S_{\delta^{\prime *} \eta} y=\int d t \eta^{\prime}(t) S_{t} y=H S_{\eta} y .
$$

Thus $S_{\delta^{\prime}}=H$ on $\mathscr{X}_{\text {reg }}(S)$ and consequently by $S_{\delta^{\prime}}=H$. But by part a one has $S_{\delta^{\prime}} S_{\delta^{\prime}}=S_{\delta^{\prime} * \delta^{\prime}}$ so $S_{\delta^{\prime} * \delta^{\prime}}=H^{2}$. By iteration of this argument one obtains the desired result for all integer $n \geq 1$.

Next we turn to the problem of identifying the fractional power $H^{\alpha}$ as defined above with the traditional definitions (see, for example, [22, Section IX.11]). We first emphasize, however, that our definition of the generator $H$ of the semigroup $S$ corresponds to the formal relation $S_{t}=\exp \{-t H\}$. Other authors, for example Yosida [22], adopt a convention which replaces $H$ by $-H$ and is consistent with the relation $S_{t}=\exp \{t H\}$. This can lead to confusion in comparisons with the literature.

The original notion of fractional power of a semigroup generator is due to Bochner [5] who observed that if $0<\alpha<1$ then there exists a family of positive functions $\left\{\mu_{t}^{\alpha}\right\}_{t \geq 0}$ on $\mathbf{R}_{+}$such that

$$
e^{-t \lambda^{\prime \prime}}=\int_{0}^{\infty} d s \mu_{t}^{\alpha}(s) e^{-\lambda s}, \quad \lambda \geq 0 .
$$

The $\mu_{t}^{\alpha}$ form a convolution semigroup (see, for details of such semigroups, [4]), for example,

$$
\mu_{t_{1}}^{\alpha} * \mu_{t_{2}}^{\alpha}=\mu_{t_{1}+t_{2}}^{\alpha}
$$

and the bounded $\sigma(\mathscr{X}, \mathscr{F})$-continuous operators

$$
S_{t}^{\alpha}=S_{\mu_{t}^{\alpha}}=\int_{0}^{\infty} d s \mu_{t}^{\alpha}(s) S_{s}
$$

form a $\sigma(\mathscr{Z}, \mathscr{F})$-continuous semigroup $S^{\alpha}$. The generator $H_{\alpha}$ of this semigroup is a natural candidate for the $\alpha$ th power of $H$ and in fact it is equal to $H^{\alpha}$ as defined above. This equality is a simple consequence of Proposition 3.3. 
In order to obtain the identification $H_{\alpha}=H^{\alpha}$ we introduce $\left\{\varphi_{t}^{\alpha}\right\}_{t} \geq 0$ as the family of $\mathbf{R}_{+}$-summable measures

$$
d \varphi_{t}^{\alpha}(s)=\left(\delta(s)-\mu_{t}^{\alpha}(s)\right) d s / s
$$

then by definition $H_{\alpha}$ is the $\sigma(\mathscr{X}, \mathscr{F})-\sigma(\mathscr{X}, \mathscr{F})$-closed operator with domain

$$
D\left(H_{\alpha}\right)=\left\{x \in X ; \sigma(\mathscr{X}, \mathscr{F})-\lim S_{\varphi_{t}^{\alpha}} x \text { exists }\right\}
$$

and with action

$$
H_{\alpha} x=\sigma(\mathscr{X}, \mathscr{F})-\lim S_{\varphi_{t}^{*}} x, \quad x \in D\left(H_{\alpha}\right) .
$$

But it is easily checked that $\varphi_{t}^{\alpha} * \eta$ is $L_{1}$-convergent to $\delta^{\alpha} * \eta$ for a nonzero $\eta \in C_{00}^{\infty}\left(\boldsymbol{R}_{+}\right)$as $t \rightarrow 0+;$ in fact, for all $\eta \in C_{00}^{\infty}\left(\boldsymbol{R}_{+}\right)$. This can be established either by explicit estimation or by observing that

$$
(t, \eta) \mapsto \mu_{t}^{\alpha}(\eta)=\int d s \mu_{t}^{\alpha}(s) \eta(s)
$$

defines a strongly continuous contraction semigroup on $C_{0}\left(\mathbf{R}_{+}\right)$whose generator acts by convolution with $\delta^{\alpha}$ (see, for example, [4]). Therefore $H_{\alpha}=S_{\delta^{\prime \prime}}^{0}$ in the notation of Proposition 3.3, as a result of this proposition and Remark 3.4. But this implies $S_{\delta^{n}}^{0}$ is $\sigma(\mathscr{X}, \mathscr{F})-\sigma(\mathscr{X}, \mathscr{F})$-closed and hence

$$
H_{\alpha}=S_{\delta^{\prime \prime}}^{0}=H^{\alpha}
$$

by Proposition 3.3.

Our next result is a strengthening of Proposition 3.3 which allows us to obtain other characterizations of $H^{\alpha}$.

THEOREM 5.4. Let $S$ be a uniformly bounded, locally $\tau(\mathscr{X}, \mathscr{F})$-equicontinuous, $\sigma(\mathscr{X}, \mathscr{F})$-continuous semigroup with generator $H$. Further let $\varphi_{j}$ be a bounded net of finite measures on $[0, \infty)$ with the property that $\varphi_{j} * \eta$ is $L_{1}$-convergent to $\delta^{\alpha} * \eta$ for each $\eta \in C_{00}^{\infty}\left(\mathbf{R}_{+}\right)$. Next for $\alpha>0$ define $W_{\alpha}$ by $W_{\alpha}(t)=\theta(t) t^{\alpha-1} e^{-t}, t \geq 0$, and assume

1. $\left\{\varphi_{j} * W_{\alpha}\right\}$ is bounded in $L_{1}\left(\mathbf{R}_{+}\right)$,

2. $\lim _{r \rightarrow \infty} \int_{r}^{\infty} d t\left|\left(\varphi_{j} * W_{\alpha}\right)(t)\right|=0$ uniformly in $j$.

Then the following conditions are equivalent for $x \in X$;

1. $x \in D\left(H^{\alpha}\right)$,

2. $S_{\varphi_{i}} x$ is $\sigma(\mathscr{Z}, \mathscr{F})$-convergent,

3. $S_{\varphi_{j}} x$ is $\tau(\mathscr{Z}, \mathscr{F})$-convergent, and if these conditions are satisfied then

$$
H^{\alpha} x=\sigma(\mathscr{Z}, \mathscr{F})-\lim S_{\varphi_{j}} x=\tau(\mathscr{X}, \mathscr{F})-\lim S_{\varphi_{j}} x .
$$


Proof. $3 \Rightarrow 2$ is evident and $2 \Rightarrow 1$ by Proposition 3.3 .

$1 \Rightarrow 3$. First remark that $D\left(H^{\alpha}\right)=D\left((I+H)^{\alpha}\right)=R\left((I+H)^{-\alpha}\right)$ and since $(I+H)^{-\alpha}$ is a constant multiple of $S_{W_{r}}$ it suffices to prove $\tau(\mathscr{Z}, \mathscr{F})$ convergence of $S_{\varphi}, S_{W_{n}} x$ for all $x \in \mathscr{Z}$. But $S_{\varphi_{j}} S_{W_{r}} S_{\eta}=S_{\varphi_{j} * W_{n} * \eta}$ for all $\eta \in$ $C_{00}^{\infty}\left(\mathbf{R}_{+}\right)$and since $\varphi_{\eta}$ is $L_{1}$-convergent to $\delta^{\alpha} * \eta$ for each $\eta \in C_{00}^{\infty}\left(\mathbf{R}_{+}\right)$it follows immediately that $S_{\varphi_{j}} S_{W_{i}} x$ is $\tau(\mathscr{X}, \mathscr{F})$-convergent to $H^{\alpha} S_{W_{\alpha}} x$ for all $x \in$ $\mathscr{X}_{\text {reg }}(s)$. But $\mathscr{Z}_{\text {reg }}(s)$ is $\sigma(\mathscr{X}, \mathscr{F})$-dense and hence $\tau(\mathscr{X}, \mathscr{F})$-dense. Moreover $S_{\varphi_{j}} S_{W_{r}}=S_{\varphi_{j} * W_{n}}$ by Proposition 3.2 and $\left\{S_{\varphi_{j} * W_{n}}\right\}$ is an equicontinuous family by Proposition 2.6, and conditions 1 and 2 on the $\varphi_{j}$. Therefore $S_{\varphi_{j}} S_{W_{i}} x$ is $\tau(\mathscr{X}, \mathscr{F})$-convergent to $H^{\alpha} S_{W_{u}} x$ for all $x \in \mathscr{Z}$ by a standard $3 \varepsilon$-argument, as used to prove Proposition 2.3.

REMARK 5.5. If $\left\{S_{t}\right\}_{t \geq 0}$ is equicontinuous, condition 2 on the $\varphi_{j}$ is not necessary.

In the sequel we give two applications of Theorem 5.4 which both arise from a special type of approximating measure. Hence as a preliminary we prove the following.

Proposition 5.6. Let $\psi$ be a finite measure on $\mathbf{R}_{+}$with Fourier transform $\hat{\psi}$ satisfying $\hat{\psi}(0)=1$ and let $\varepsilon_{j}>0$ be a net converging to zero. For $\alpha>0$ define $\varphi_{j}$ as the bounded net of finite measures with Fourier transforms

$$
\hat{\varphi}_{j}(\lambda)=(-\lambda)^{\alpha} \hat{\psi}\left(\varepsilon_{j} \lambda\right)
$$

Then $\varphi_{j}$ satisfies all the hypotheses of Theorem 5.4.

Proof. Let $\psi_{j}$ denote the inverse Fourier transform of $\lambda \mapsto \hat{\psi}\left(\varepsilon_{j} \lambda\right)$. Then $\varphi_{j}=\psi_{j} * \eta$ is $L_{1}$-convergent to $\delta^{\alpha} * \eta$ for each $\eta \in C_{00}^{\infty}\left(\mathbf{R}_{+}\right)$if $\psi_{j} * \xi$ is $L_{1}$-convergent to $\xi$ for each infinitely-often differentiable $\xi \in L_{1}\left(\mathbf{R}_{+}\right)$. But

$$
\int_{0}^{\infty} d s\left|\left(\psi_{j} * \xi\right)(s)-\xi(s)\right|=\int_{0}^{\infty} d s\left|\int_{0}^{\infty} d t \psi(t)\left(\xi\left(s-\varepsilon_{j} t\right)-\xi(s)\right)\right|
$$

because $\psi$ has total integral one. The desired convergence then follows from the Lebesgue dominated convergence theorem.

Next, to obtain the boundedness properties of the $\varphi_{j}$, we note that $\varphi_{j} *$ $W_{\alpha}=\psi_{j} * X_{\alpha}$ where $X_{\alpha}=\delta+Y_{\alpha}$ and $Y_{\alpha}$ is the inverse Fourier transform of $(-i \lambda /(1-i \lambda))^{\lambda}-1$. But then $Y_{\alpha}$ is an integrable function by Proposition 4.5. conditions 1 and 2 of Theorem 5.4 follow immediately.

As a first application of these application results we derive the characterization of $H^{\alpha}$ by the integral transform described in the introduction. The last necessary step for this characterization is the following. 
Proposition 5.7. Let $\alpha>0$ and $m$ an integer strictly greater than $\alpha$. Then there is an integrable function $\psi_{\alpha, m}$ such that

$$
\int_{\varepsilon}^{\infty} \frac{d t}{t}{\frac{\left(1-e^{i \lambda t}\right)^{m}}{t^{\alpha}}}^{m}=\lambda^{\alpha} \hat{\psi}_{\alpha, m}(\alpha \lambda)
$$

for all $\varepsilon>0$ and all $\lambda$ in the upper half-plane.

Proof. The left-hand side of $(*)$ is analytic in the upper half-plane, and the integral can be regarded as a contour integral along the positive real axis from $\varepsilon$ to $\infty$. Take $\lambda$ in the upper half plane, and change variable of integration from $t$ to $s=\lambda t$. This gives:

$$
\int_{\varepsilon}^{\infty} \frac{d t}{t}{\frac{\left(1-e^{i \lambda t}\right)^{m}}{t^{\alpha}}}^{m}=\lambda^{\alpha} \int_{\gamma(\varepsilon, \lambda)} \frac{d s}{s}{\frac{\left(1-e^{i s}\right)^{m}}{s^{\alpha}}}^{m}
$$

where $\gamma(\varepsilon, \lambda)$ is the contour running from $\varepsilon \lambda$ to $\infty$ along the ray from the origin through $\lambda$. Because the integrand is well behaved in the upper halfplane, it follows from the Cauchy Integral Theorem that

$$
\int_{\gamma(\varepsilon, \lambda)} \frac{d s}{s}{\frac{\left(1-e^{i s}\right)^{m}}{s^{\alpha}}}^{m}
$$

depends only on the starting point $\varepsilon \lambda$ of the contour of integration and not on $\varepsilon$ and $\lambda$ separately. In other words, we can write

$$
\int_{\gamma(\varepsilon, \lambda)} \frac{d s}{s}{\frac{\left(1-e^{i s}\right)^{m}}{s^{\alpha}}}^{m}=\hat{\psi}_{\alpha, m}(\varepsilon \lambda)
$$

with $\hat{\psi}_{\alpha, m}$ analytic in the upper half-plane. It remains to be proved that $\hat{\psi}_{\alpha, m}$ is the Fourier transform of an integrable function.

Introduce $\hat{\chi}$ by

$$
\hat{\chi}(\lambda)=\frac{d}{d \lambda} \hat{\psi}_{\alpha, m}(\lambda)=-\left(1-e^{i \lambda}\right)^{m} / \lambda^{\alpha+1}
$$

Since $\hat{\chi}$ is integrable it is the Fourier transform of a continuous function $\chi$. In fact since $\lambda \mapsto \hat{\chi}(\lambda)(1+|\lambda|)^{\varepsilon}$ is integrable for sufficiently small positive $\varepsilon$, it follows that $\chi$ is actually Hölder continuous. Moreover, it is easily checked from the definition that $\hat{\psi}_{\alpha, m}(\lambda) \rightarrow 0$ as $\lambda \rightarrow \infty$, so $\int_{-\infty}^{\infty} \hat{\chi}(\lambda) d \lambda=0$, that is, $\chi(0)=0$.

Finally $\hat{\chi}$ is a smooth function of $\lambda$ except at zero, where it can be written, locally, as the product of $\lambda^{m-1-\alpha}$ by an analytic function. Hence, by Corollary 4.2, $\chi(t)=O\left(|t|^{-m+\alpha}\right)$ at infinity.

Combining all those considerations, we see that $t \mapsto \chi(t) /(-i t)$ is an integrable function whose Fourier transform has the same derivative in the sense of distributions as $\hat{\psi}_{\alpha, m}(\lambda)$. Thus, $\hat{\psi}_{\alpha, m}(\lambda)$ and the Fourier transform 
of $t \mapsto \chi(t) /(-i t)$ differ by a constant. Since they both vanish as $\lambda \rightarrow \infty$, this constant must be 0 , that is, $\hat{\psi}_{\alpha, m}(\lambda)$ is the Fourier transform of the integrable function $t \mapsto \chi(t) /(-i t)$.

Now combining Theorem 5.4 with Propositions 5.6 and 5.7 one draws the following conclusion.

THEOREM 5.8. Let $S$ be a uniformly bounded, locally $\tau(\mathscr{X}, \mathscr{F})$-equicontinuous, $\sigma(\mathscr{X}, \mathscr{F})$-continuous semigroup with generator $H$. Further, for $\alpha>0$, let $m$ be an integer strictly greater than $\alpha$.

Then the following conditions are equivalent, for $x \in \mathscr{Z}$;

1. $x \in D\left(H^{\alpha}\right)$,

2. $\sigma(\mathscr{Z}, \mathscr{F})-\lim \int_{\varepsilon}^{\infty} \frac{d t}{t}{\frac{\left(I-S_{t}\right)^{m}}{t^{n}}}^{m} x$ exists,

3. $\tau(\mathscr{X}, \mathscr{F})-\lim \int_{\varepsilon}^{\infty} \frac{d t}{t}{\frac{\left(I-S_{1}\right)}{t^{n}}}^{m} x$ exists, and if these conditions are satisfied then

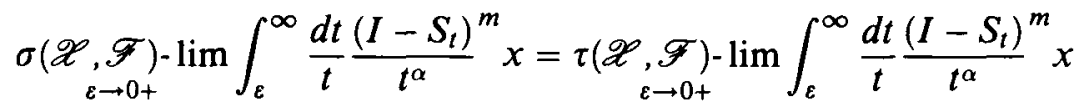

$$
\begin{aligned}
& =c_{\alpha, m} H^{\alpha} x
\end{aligned}
$$

where

$$
c_{\alpha, m}=\int_{0}^{\infty} \frac{d t}{t}{\frac{\left(1-e^{-t}\right)^{m}}{t^{\alpha}}}
$$

Proof. This follows from the foregoing results because

$$
\int_{\varepsilon}^{\infty} \frac{d t}{t}{\frac{\left(1-S_{t}\right)^{m}}{t^{\alpha}}}^{m}=\int_{0}^{\infty} d s S_{s}\left(\int_{\varepsilon}^{\infty} \frac{d t}{t^{1+\alpha}} \sum_{p=0}^{m}\left(\begin{array}{c}
m \\
p
\end{array}\right)(-1)^{p} \delta(s-p t)\right)=S_{\varphi_{a, m . \varepsilon}}
$$

where $\varphi_{\alpha, m, \varepsilon}$ is the inverse Fourier transform of

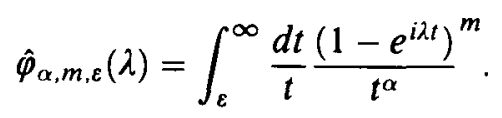

Finally we derive the formula $\left(H^{\alpha}\right)^{\beta}=H^{\alpha \beta}$ for $0<\alpha<1$ and $\beta>0$.

THEOREM 5.9. Let $S$ be a uniformly bounded, locally $\tau(\mathscr{X}, \mathscr{F})$-equicontinuous, $\sigma(\mathscr{X}, \mathscr{F})$-continuous semigroup with generator $H$ and let $0<$ $\alpha<1$.

\section{It follows that}

1. $H^{\alpha}$ generates a uniformly bounded, locally $\tau(\mathscr{Z}, \mathscr{F})$-equicontinuous, $\sigma(\mathscr{X}, \mathscr{F})$-continuous semigroup $S^{\alpha}$,

2. if $\beta>0$ then

$$
\left(H^{\alpha}\right)^{\beta}=H^{\alpha \beta} .
$$


Proof. 1. We have already mentioned prior to Theorem 5.4 that $H^{\alpha}$ generates a $\sigma(\mathscr{Z}, \mathscr{F})$-continuous semigroup $S^{\alpha}$ where

$$
S_{t}^{\alpha}=\int_{0}^{\infty} d s \mu_{t}^{\alpha}(s) S_{s}
$$

and the $\left\{\mu_{t}^{\alpha}\right\}_{t \geq 0}$ form a convolution semigroup. This is basically Bochner's observation. The $\mu_{t}^{\alpha}$ can be identified as the inverse Fourier transforms of the functions $\lambda \mapsto \exp \left\{-t(-i \lambda)^{\alpha}\right\}$ and their most important properties are

$$
\begin{gathered}
\mu_{t}^{\alpha}(s) \geq 0, \\
\int_{0}^{\infty} d s \mu_{t}^{\alpha}(s)=1, \\
\mu_{t}^{\alpha}(s)=t^{-1 / \alpha} \mu_{1}^{\alpha}\left(s t^{-1 / \alpha}\right) .
\end{gathered}
$$

The second and third properties are easily derived but the positivity property is less obvious. It follows because $\lambda \mapsto \lambda^{\alpha}$ is a Bernstein function (see [22] or [4]). Note that the $\sigma(\mathscr{Z}, \mathscr{F})$-continuity of $S^{\alpha}$ then follows because

$$
f\left(S_{t}^{\alpha} x\right)=\int_{0}^{\infty} d s \mu_{1}^{\alpha}(s) f\left(S_{s t^{1 / \mu}} x\right) .
$$

Moreover if $0<t \leq 1$ then

$$
\int_{r}^{\infty} d s \mu_{r}^{\alpha}(s)=\int_{r t-1 / n}^{\infty} d s \mu_{1}^{\alpha}(s) \leq \int_{r}^{\infty} d s \mu_{1}^{\alpha}(s)
$$

because of properties (1) and (3) of the $\mu_{t}^{\alpha}$. Hence

$$
\lim _{r \rightarrow \infty} \int_{r}^{\infty} d s \mu_{t}^{\alpha}(s)=0
$$

uniformly for $t \in(0,1]$ by properties (1) and (2). Therefore $S_{t}^{\alpha}=S_{\mu_{t}^{\alpha}}$ is locally equicontinuous by Proposition 2.6.

2. Since $H^{\alpha}$ generates $S^{\alpha}$ it follows from Theorem 5.8 that $\left(H^{\alpha}\right)^{\beta}$ is proportional to

$$
\lim _{\varepsilon \rightarrow 0+} \int_{\varepsilon}^{\infty} \frac{d t}{t}{\frac{\left(I-S_{t}^{\alpha}\right)^{m}}{t^{\beta}}}^{m}
$$

where $m$ is an integer strictly larger than $\beta$ and where the limit can be taken either in the $\sigma(\mathscr{X}, \mathscr{F})$-, or the $\tau(\mathscr{X}, \mathscr{F})$-, topology. But since $S_{t}^{\alpha}=S_{\mu_{t}^{\alpha}}$ and $\mu_{t}^{\alpha}$ is the inverse Fourier transform of $\lambda \mapsto \exp \left\{-t(-i \lambda)^{\alpha}\right\}$ it follows that

$$
\int_{\varepsilon}^{\infty} \frac{d t}{t}{\frac{\left(I-S_{l}^{\alpha}\right)^{m}}{t^{\beta}}}^{m}=S_{\varphi_{\text {s. . . . . . }}}
$$

where $\varphi_{\alpha, \beta, m, \varepsilon}$ is the inverse Fourier transform of

$$
\hat{\varphi}_{\alpha, \beta, m, \varepsilon}(\lambda)=\int_{0}^{\infty} \frac{d t}{t} \frac{\left(1-e^{-t(-i \lambda)^{\alpha}}\right)^{m}}{t^{\beta}} .
$$


As in the proof of Proposition 5.7, by changing integration variable from $t$ to $s=\lambda t^{1 / \alpha}, \hat{\varphi}_{\alpha, \beta, m, \varepsilon}$ can be written as

$$
\hat{\varphi}_{\alpha, \beta, m, \varepsilon}(\lambda)=\lambda^{\alpha \beta} \hat{\psi}_{\alpha, \beta, m}\left(\varepsilon^{1 / \alpha} \lambda\right)
$$

with $\hat{\psi}_{\alpha, \beta, m}$ analytic in the upper half-plane and

$$
\hat{\psi}_{\alpha, \beta, m}(\lambda)=\alpha \int_{\lambda}^{\infty} \frac{d s}{s} \frac{\left(1-e^{-(-i s)^{\alpha}}\right)^{m}}{s^{\alpha \beta}} .
$$

Then arguing as in Proposition 5.7 one deduces that $\hat{\psi}_{\alpha, \beta, m}$ is the Fourier transform of an integrable function $\psi_{\alpha, \beta, m}$. Hence by Proposition 5.6 and Theorem 5.5 one concludes that the $\sigma(\mathscr{X}, \mathscr{F})$-limit of $S_{\varphi_{\alpha, \beta, m, \ell}} x$ as $\varepsilon \rightarrow 0+$ exists if, and only if, $x \in D\left(H^{\alpha \beta}\right)$ and then the limit is proportional to $H^{\alpha \beta} x$. This establishes that $\left(H^{\alpha}\right)^{\beta}$ is proportional to $H^{\alpha \beta}$ but it is easily concluded that the factor of proportionality is one.

The foregoing results apply to the special cases of strongly continuous and weakly* continuous semigroups and in both these cases the results can be strengthened. We conclude this section with some comments on these special cases.

Strong continuity of a semigroup $S$ is equivalent to weak, or $\sigma(\mathscr{X}, \mathscr{F})$-, continuity. Thus to discuss strongly continuous semigroups we choose $\mathscr{F}=$ $\mathscr{X}^{*}$. But the $\tau\left(\mathscr{X}, \mathscr{X}^{*}\right)$-topology coincides with the norm topology and equicontinuity of $S$ is equivalent to uniform boundedness. Hence Theorem 5.4, combined with Remark 5.5, gives the following statement. Let $S$ be a uniformly bounded, strongly continuous, semigroup with generator $H$. Further let $\varphi_{j}$ be a bounded net of finite measures on $[0, \infty)$ with the property that $\varphi_{j} * \eta$ is $L_{1}$-convergent to $\delta^{\alpha} * \eta$ for each $\eta \in C_{00}^{\infty}\left(\mathbf{R}_{+}\right)$and $\left\{\varphi_{j} * W_{\alpha}\right\}$ is bounded to $L_{1}\left(\mathbf{R}_{+}\right)$, where $\alpha>0$ and $W_{\alpha}(t)=\theta(t) t^{\alpha-1} e^{-t}, t \geq 0$. Then

$$
H^{x}=\mathrm{w} \cdot \lim _{j} S_{\varphi_{j}} x=\mathrm{s} \cdot \lim _{j} S_{\varphi_{j}} x
$$

where the limits exist if, and only if, $x \in D\left(H^{\alpha}\right)$. In particular one may choose the $\varphi_{j}$ in the manner of Propositions 5.6 and 5.7 and hence obtain the analogue of Theorem 5.8,

$$
c_{\alpha, m} H^{\alpha} x=\mathrm{w}_{\varepsilon \rightarrow 0} \lim _{\varepsilon} \int_{\varepsilon}^{\infty} \frac{d t}{t}{\frac{\left(I-S_{t}\right)^{m}}{t^{\alpha}}}^{m}=\mathrm{s}_{\varepsilon \rightarrow 0} \lim _{\varepsilon} \int_{\varepsilon}^{\infty} \frac{d t}{t}{\frac{\left(I-S_{t}\right)^{m}}{t^{\alpha}}}^{m}
$$

where the limits exist if, and only if, $x \in D\left(H^{\alpha}\right)$. This last statement corresponds to the definition of $H^{\alpha}$ given by Balakrishnan [2] subsequently developed in [14], [3] and [11]. Our derivation is, however quite different to the previous ones although the use of distribution theory has something in common with the Lions-Peetre approach.

Next assume $\mathscr{X}$ has a predual $\mathscr{X}_{*}$, set $\mathscr{F}=\mathscr{X}_{*}$, and consider a $\sigma\left(\mathscr{X}, \mathscr{X}_{*}\right)$ continuous semigroup $S$, that is, a weak*-continuous semigroup. It follows 
from the discussion preceding Proposition 2.4 that $S$ is locally equicontinuous, but it is not necessarily equicontinuous even if it is uniformly bounded, for example translations on $L_{\infty}$ are not $\tau\left(L_{\infty}, L_{1}\right)$-equicontinuous. Nevertheless, weak* compactness and duality arguments originating with DeLeeuw [7] can be used to improve the foregoing results. Let $S$ be a uniformly bounded $\sigma\left(\mathscr{Z}, \mathscr{X}_{*}\right)$-continuous semigroup with generator $H$. Further let $\varphi_{j}$ be a bounded net of finite measures on $[0, \infty)$ with the property that $\varphi_{j} * \eta$ is $L_{1}$-convergent to $\delta^{\alpha} * \eta$ for each $\eta \in C_{00}^{\infty}\left(\mathbf{R}_{+}\right)$and $\left\{\varphi_{j} * W_{\alpha}\right\}$ is bounded in $L_{1}\left(\mathbf{R}_{+}\right)$, where $\alpha>0$ and $W_{\alpha}(t)=\theta(t) t^{\alpha-1} e^{-t}, t \geq 0$. Then $x \in D\left(H^{\alpha}\right)$ if, and only if $\sup _{j}\left\|S_{\varphi_{j}} x\right\|<+\infty$ and if these conditions are satisfied

$$
H^{\alpha} x=\mathrm{w}^{*} \cdot \lim _{j} S_{\varphi_{j}} x .
$$

For example, $x \in D\left(H^{\alpha}\right)$ if and only if there is a $y$ such that $x=S_{W_{a}} y$ and then

$$
\sup _{j}\left\|S_{\varphi_{j}} x\right\|=\sup _{j}\left\|S_{\varphi_{j} * W_{\alpha}} y\right\| \leq \sup _{j}\left\|\varphi_{j} * W_{\alpha}\right\|_{1} \cdot\|y\| .
$$

Conversely if $\sup _{j}\left\|S_{\varphi,} x\right\|<+\infty$ then by weak*-compactness of the unit ball there is a weak ${ }^{*}$-convergent subnet $S_{\varphi^{\prime}} x$, whose limit we denote by $y$. But the adjoint semigroup $S^{*}$ is strongly continuous with generator $H^{*}$ and, by definition, $\left(H^{\alpha}\right)^{*}=\left(H^{*}\right)^{\alpha}$. Now if $z \in D\left(\left(H^{*}\right)^{\alpha}\right)$ then

$$
(y, z)=\lim _{j^{\prime}}\left(x, S_{\varphi_{j^{\prime}}}^{*} z\right)=\left(x,\left(H^{*}\right)^{\alpha} z\right)
$$

by the conclusion of the previous paragraph. Consequently $x \in D\left(H^{\alpha}\right)$ and $y=H^{\alpha} x$. Again one can make particular choices of $\varphi_{j}$ and it follows as in Theorem 5.8 that

$$
c_{\alpha, m} H^{\alpha} x=\mathrm{w}^{*} \cdot \lim _{\varepsilon \rightarrow 0} \int_{\varepsilon}^{\infty} \frac{d t}{t}{\frac{\left(I-S_{t}\right)^{m}}{t^{\alpha}}}^{m}
$$

with the limit existing if and only if $x \in D\left(H^{\alpha}\right)$. This latter result was first obtained by Berens, Butzer, Westphal [3] and independently by Komatsu [11]. Note that it also follows from Proposition 5.6 and 5.7 that this last result is also true with the weak*-limit replaced by the $\tau\left(\mathscr{X}, \mathscr{X}_{*}\right)$-limit.

\section{Fractional derivatives}

Again we consider a uniformly bounded, locally equicontinuous, $\sigma(\mathscr{X}, \mathscr{F})$ continuous semigroup $S$ with generator $H$. The aim of this section is to identify the fractional powers $H^{\alpha}$ of $H$ as fractional derivatives of $S$. Explicitly we establish that

$$
H^{\alpha} x=\lim _{t \rightarrow 0+}\left(\left(I-S_{t}\right) / t\right)^{\alpha} x
$$


where the $\sigma(\mathscr{Z}, \mathscr{F})$-, or $\tau(\mathscr{X}, \mathscr{F})$-, limit exists if, and only if, $x \in D\left(H^{\alpha}\right)$. There are a number of necessary preliminaries to the proof. First we must define the fractional powers of $\left(I-S_{t}\right) / t$.

Since $\left(I-S_{t}\right) / t$ is norm bounded, for $t>0$, one can define its exponential by a uniformly convergent power series expansion. Then

$$
\exp \left\{-s\left(I-S_{t}\right) / t\right\}=e^{-s / t} \sum_{n \geq 0} \frac{(s / t)^{n}}{n !} S_{n t}
$$

Hence if $\left\|S_{t}\right\| \leq M$ for all $t>0,\left\|\exp \left\{-s\left(I-S_{t}\right) / t\right\}\right\| \leq M$. Thus the semigroups $s \mapsto T_{s}=\exp \left\{-s\left(I-S_{t}\right) / t\right\}$ are uniformly bounded, and uniformly continuous. Therefore the fractional powers of their generators can be defined as in Section 5 .

The second step in the proof is to establish the existence of finite measures $\mu_{+}^{\alpha}$ on $\mathbf{R}_{+}$such that the fractional powers have the representation

$$
\left(\left(I-S_{t}\right) / t\right)^{\alpha}=S_{\mu_{t}^{\alpha}} .
$$

The basic result is a single operator expansion based on Theorem 5.8.

Proposition 6.1. Let $T$ be a bounded operator on the Banach space $\mathscr{X}$ with the property that $\left\|T^{n}\right\| \leq M$ for all $n=1,2, \ldots$, and some $M<+\infty$ and let $\alpha>0$. It follows that $(I-T)$ generates a uniformly bounded norm continous semigroup and

$$
\begin{aligned}
(I-T)^{\alpha} & =c_{\alpha, m}^{-1} \int_{0}^{\infty} d s s^{-1-\alpha}\left(I-e^{-s(I-T)}\right)^{m} \\
& =\sum_{n \geq 0} c_{n}^{\alpha} T^{n}
\end{aligned}
$$

where $m>\alpha$ is an integer,

$$
c_{\alpha, m}=\int_{0}^{\infty} d s s^{-1-\alpha}\left(1-e^{-s}\right)^{m},
$$

and $c_{n}^{\alpha}$ are the coefficients in the binomial expansion of $x \mapsto(1-x)^{\alpha}$.

Proof. First consider the semigroup $S$ defined by the norm convergent series

$$
S_{s}=\exp \{-s(I-T)\}=e^{-s} \sum_{n \geq 0} \frac{s^{n}}{n !} T^{n} .
$$

It follows immediately that $S$ is norm continuous, $\left\|S_{s}\right\| \leq M$ for all $s \geq 0$, and the generator of $S$ is $I-T$. Therefore $(I-T)^{\alpha}$ is defined and

$$
(I-T)^{\alpha}=c_{\alpha, m}^{-1} \int_{0}^{\infty} d s s^{-1-\alpha}\left(I-e^{-s(I-T)}\right)^{m}
$$

by Theorem 5.8 . 
Next we prove that

$$
(I-T)^{\alpha}=\sum_{n \geq 0} c_{j}^{\alpha} T^{n} .
$$

But it suffices to establish this for $0<\alpha<1$. The general case then follows by a sequence of simple observations. First it is obvious for integral $\alpha$. Second, if $\alpha=m+\beta$ with $m$ an integer and $0<\beta<1$ then $(I-T)^{\alpha}=(I-T)^{m}(I-T)^{\beta}$ by Theorem 5.3. Third, one has

$$
\sum_{n \geq 0} c_{n}^{\alpha} T^{n}=(I-T)^{n} \sum_{n \geq 0} c_{n}^{\beta} T^{n}
$$

by straightforward calculation. Therefore we now assume $0<\alpha<1$. But then

$$
(I-T)^{\alpha}=c_{\alpha, 1}^{-1} \int_{0}^{\infty} d s s^{-1-\alpha}\left(I-e^{-s} e^{s T}\right)
$$

by the first part of the proof. Consequently

$$
\begin{aligned}
(I-T)^{\alpha} & =c_{\alpha, 1}^{-1} \int_{0}^{\infty} d s s^{-1-\alpha}\left(\left(1-e^{-s}\right) I+e^{-s}\left(I-e^{s T}\right)\right) \\
& =I+c_{\alpha, 1}^{-1} \int_{0}^{\infty} d s s^{-1-\alpha} e^{-s}\left(I-e^{s T}\right)
\end{aligned}
$$

Now substitution of the Taylor's series expansion of $\left(I-e^{s T}\right)$ gives a series expansion for $(I-T)^{\alpha}$ and the coefficients of this expansion are

$$
c_{\alpha, 1}^{-1} \Gamma(n-\alpha) / n !
$$

But for large enough $n$, for example, for $n>\alpha+1$, one finds

$$
0<\Gamma(n-\alpha) / n ! \leq\left(e^{2} / n\right)^{1+\alpha}
$$

by an estimate of the Stirling type. Moreover $\left\|T^{n}\right\| \leq M$. Hence the expansion

$$
(I-T)^{\alpha}=I+c_{\alpha, 1}^{-1} \sum_{n \geq 1}(\Gamma(n-\alpha) / n !) T^{n}
$$

is norm convergent. But since this is valid for the operator $T$ of multiplication by $t$, with $|t| \leq 1$. on $\mathbb{C}$ one must have $c_{\alpha, 1}{ }^{-1} \Gamma(n-\alpha) / n !=c_{n}^{\alpha}$. Hence

$$
(I-T)^{\alpha}=I+\sum_{n \geq 1} c_{n}^{\alpha} T^{n}
$$

COROLlARY 6.2. Let $\mu_{t}^{\alpha}$ denote the inverse Fourier transform of the function $\lambda \mapsto\left(\left(1-e^{i \lambda t}\right) / t\right)^{\alpha}$. Then

$$
\left(\left(I-S_{t}\right) / t\right)^{\alpha}=S_{\mu_{t}^{\prime \prime}}
$$


Proof. It follows from Proposition 6.1, applied with $T=S_{l}$, that

$$
\left(\left(I-S_{t}\right) / t\right)^{\alpha}=\int_{0}^{\infty} d s S_{s}\left(\sum_{n \geq 0} c_{n}^{\alpha} \delta(s-n t) / t^{\alpha}\right) .
$$

But

$$
\int_{-\infty}^{\infty} d s e^{i \lambda s}\left(\sum_{n \geq 0} c_{n}^{\alpha} \delta(s-n t) / t^{\alpha}\right)=\left(\left(1-e^{i \lambda t}\right) / t\right)^{\alpha}
$$

The desired result on fractional derivatives now follows from the results of Sections 4 and 5.

THEOREM 6.3. Let $S$ be a uniformly bounded, locally $\tau(\mathscr{X}, \mathscr{F})$-equicontinuous, $\sigma(\mathscr{X}, \mathscr{F})$-continuous semigroup with generator $H$. Let $\alpha>0$.

Then the following conditions are equivalent for $x \in \mathscr{X}$;

1. $x \in D\left(H^{\alpha}\right)$,

2. $\sigma(\mathscr{X}, \mathscr{F})-\lim _{t \rightarrow 0+}\left(\left(I-S_{t}\right) / t\right)^{\alpha} x$ exists,

3. $\tau(\mathscr{X}, \mathscr{F})-\lim _{t \rightarrow 0+}\left(\left(I-S_{t}\right) / t\right)^{\alpha} x$ exists, and if these conditions are satisfied then

$$
\begin{aligned}
\sigma(\mathscr{X}, \mathscr{F})-\lim \left(\left(I-S_{t}\right) / t\right)^{\alpha} x & =\tau(\mathscr{X}, \mathscr{F})-\lim \left(\left(I-S_{t}\right) / t\right)^{\alpha} x \\
& =H^{\alpha} x .
\end{aligned}
$$

Proof. By Corollary 6.2 one has

$$
\left(\left(I-S_{t}\right) / t\right)^{\alpha}=S_{\mu_{t}^{\alpha}}
$$

where $\mu_{t}^{\alpha}$ is the inverse Fourier transform of $\lambda \mapsto \hat{\mu}_{t}^{\alpha}(\lambda)=\left(\left(1-e^{i \lambda t}\right) / t\right)^{\alpha}$. But then $\hat{\mu}_{t}^{\alpha}(\lambda)=(-i \lambda)^{\alpha} \hat{\psi}(t \lambda)$ where $\hat{\psi}(\lambda)=\left(\left(1-e^{i \lambda}\right) /(-i \lambda)\right)^{\alpha}$. Now it follows from Proposition 4.5 that $\hat{\psi}$ is the inverse Fourier transform of an integrable function $\psi$, and clearly $\hat{\psi}(0)=1$. Therefore it follows from Proposition 5.6 that for each net $t_{j}>0$ converging to zero the finite measures $\varphi_{j}=\mu_{t_{j}}^{\alpha}$ satisfy the hypotheses of Theorem 5.4. The deisred result follows as an immediate corollary of this latter theorem.

Finally we note that this result applies immediately to uniformly bounded, strongly, or weakly*, continuous semigroups by the discussion at the end of Section 5 . 


\section{References}

[1] W. Arveson, 'On groups of automorphisms of operator algebras', J. Funct. Anal. 15 (1974), 217-243.

[2] A. V. Balakrishnan, 'An operational calculus for infinitesimal generators of semigroups', Trans. Amer. Math. Soc. 91 (1959). 330-353.

A. V. Balakrishnan, 'Fractional powers of closed operators and the semigroups generated by them', Pacific J. Math. 10 (1960), 419-437.

[3] H. Berens, P. L. Butzer, and U. Westphal, 'Representation of fractional powers of infinitesimal generators of semigroups', Bull. Amer. Math. Soc. 74 (1968), 191-196.

[4] C. Berg and G. Forst, Potential theory on locally compact abelian groups (Springer-Verlag, 1975).

[5] S. Bochner, 'Diffusion equations and stochastic processes', Proc. Nat. Acad. Sci. U.S.A. 35 (1949), 369-370.

[6] O. Bratteli and D. W. Robinson, Operator algebras and quantum statistical mechanics. I (Springer-Verlag, 1979).

[7] $\mathrm{K}$. de Leeuw, 'On the adjoint semigroup and some problems in the theory of approximation', Math. Z. 73 (1960), 219-234.

[8] N. Dunford and J. T. Schwartz, Linear operators. I (Interscience, 1958).

[9] A. Friedman, Partial differential equations (Holt, Reinhart and Winston, New York, 1969).

[10] T. Kato, 'Note on fractional powers of linear operators', Proc. Japan Acad. 36 (1960), 94-96.

[10] T. Kato, 'Fractional powers of dissipative operators', J. Math. Soc. Japan 13 (1961), 246274.

[11] H. Komatsu, 'Fractional powers of operators', Pacific J. Math. 19 (1966), 285-346.

H. Komatsu, 'Fractional powers of operators. II. Interpolation spaces', Pacific J. Math. 21 (1967), 89-111.

H. Komatsu, 'Fractional powers of operators. III. Negative powers', J. Math. Soc. Japan 21 (1969), 205-220.

H. Komatsu, 'Fractional powers of operators. IV. Potential operators', J. Math. Soc. Japan 21 (1969), 221-228.

H. Komatsu, 'Fractional powers of operators. V. Dual Operators', J. Fac. Sci. Univ. Tokyo 17 (1970), 373-396.

[12] M. A. Krasnoselskii and P. E. Sobolevskii, 'Fractional powers of operators defined on Banach space', Dokl. Akad. Nauk SSSR 129 (1959), 499-502.

[13] M. A. Krasnoseiskii, P. D. Zabreiko, E. I. Pustylnik, and P. E. Sobolevskii, Integral operators in spaces of summable functions (Noordhoff, Leiden, 1976).

[14] J. L. Lions, and J. Peetre, 'Sur une classe d'espaces d'interpolation', Inst. Hautes Études Sci. Publ. Math. 19 (1964), 5-68.

[15] E. Nelson, 'A functional calculus using singular Laplace integrals', Trans. Amer. Math. Soc. 88 (1958), 400-413.

[16] A. Pazy, Semigroups of linear operators and applications to partial differential equations (Springer-Verlag, 1983).

[17] R. S. Phillips, 'On the generation of semi-groups of linear operators', Pacific J. Math. 2 (1952), 343-369.

[18] L. Schwartz, Lectures on mixed problems in partial differential equations and the representation of semigroups (Tata Inst. of Fund. Res., Bombay, 1958).

[19] H. Tanabe, Equations of evolution (Pitman, London, 1979). 
[20] H. Triebel, Interpolation theory, function spaces, differential operators (North-Holland, 1978).

[21] J. Watanabe, 'On some properties of fractional powers of linear operators, Proc. Japan Acad. 37 (1961), 273-275.

[22] K. Yosida, Functional analysis (Springer-Verlag, 1974).

\section{IHES}

91440 Bures-sur-Yvette

France
Mathematics Department Institute of Advanced Studies Australian National University Canberra Australia 